\title{
A microstructural approach to bed load transport: mean behaviour and fluctuations of particle transport rates
}

\author{
C. Ancey $\dagger$ and J. Heyman \\ Environmental Hydraulics Laboratory, École Polytechnique Fédérale de Lausanne, 1015 Lausanne, \\ Switzerland
}

(Received 22 July 2013; revised 24 January 2014; accepted 31 January 2014; first published online 10 March 2014)

This paper concerns a model of bed load transport, which describes the advection and dispersion of coarse particles carried by a turbulent water stream. The challenge is to develop a microstructural approach that, on the one hand, yields a parsimonious description of particle transport at the microscopic scale and, on the other hand, leads to averaged equations at the macroscopic scale that can be consistently interpreted in light of the continuum equations used in hydraulics. The cornerstone of the theory is the proper determination of the particle flux fluctuations. Apart from turbulence-induced noise, fluctuations in the particle transport rate are generated by particle exchanges with the bed consisting of particle entrainment and deposition. At the particle scale, the evolution of the number of moving particles can be described probabilistically using a coupled set of reaction-diffusion master equations. Theoretically, this is interesting but impractical, as solving the governing equations is fraught with difficulty. Using the Poisson representation, we show that these multivariate master equations can be converted into Fokker-Planck equations without any simplifying approximations. Thus, in the continuum limit, we end up with a Langevin-like stochastic partial differential equation that governs the time and space variations of the probability density function for the number of moving particles. For steady-state flow conditions and a fixed control volume, the probability distributions of the number of moving particles and the particle flux can be calculated analytically. Taking the average of the microscopic governing equations leads to an average mass conservation equation, which takes the form of the classic Exner equation under certain conditions carefully addressed in the paper. Analysis also highlights the specific part played by a process we refer to as collective entrainment, i.e. a nonlinear feedback process in particle entrainment. In the absence of collective entrainment the fluctuations in the number of moving particles are Poissonian, which implies that at the macroscopic scale they act as white noise that mediates bed evolution. In contrast, when collective entrainment occurs, large non-Poissonian fluctuations arise, with the important consequence that the evolution at the macroscopic scale may depart significantly that predicted by the averaged Exner equation. Comparison with experimental data gives satisfactory results for steady-state flows.

Key words: particle/fluid flows, river dynamics, sediment transport 


\section{Introduction}

The objective of this paper is to present a basic microstructural approach to bed load transport, which has a firmer physical basis than the common empirical relationships and helps clarify a number of points related to the huge variability (often more than one order of magnitude) observed in particle flux time series. The core of the theory is thus the modelling of fluctuations in a physical system comprising coarse particles carried by a turbulent water stream.

There is no doubt that, merely as a result of water turbulence and varying sediment availability, sediment transport exhibits fluctuations. The key is to identify the relevance of these fluctuations in the description of sediment transport at a macroscopic scale. In other words, is bed load transport a noise-driven physical system, for which the macroscopic features cannot be properly described using the averaged equations? Or, on the contrary, is the macroscopic behaviour only marginally affected by fluctuations, therefore indicating that a description either within the framework of continuum mechanics or using empirical relationships between macroscopic variables is reasonable?

To better understand the background of this question, let us first make an analogy with the Reynolds-averaged Navier-Stokes equations. When using the Reynolds decomposition, all of the flow variables are separated into time-averaged and fluctuating parts. For the velocity field $\boldsymbol{u}=\overline{\boldsymbol{u}}+\boldsymbol{u}^{\prime}$, with $\overline{\boldsymbol{u}}$ the time-averaged velocity and $\boldsymbol{u}^{\prime}$ the fluctuations, the decomposition gives rise to the Reynolds stress tensor $\sigma_{R}=-\overline{\varrho \boldsymbol{u}^{\prime} \boldsymbol{u}^{\prime}}$. With increasing Reynolds numbers, the fluctuating part of the velocity can no longer be discarded relative to the mean velocity and the Reynolds stress tensor starts to play a prominent part in the momentum conservation equation. In the simplest cases, algebraic closure equations (e.g. Prandtl's mixing length model) can be used to relate the components of the Reynolds stress tensor $\sigma_{R}$ to some of the mean viscous stress tensor components, and therefore the structure of the original Navier-Stokes equations is retained. In more realistic cases, the Navier-Stokes equations no longer form an independent set of equations, but depend on additional (non-algebraic) closure equations, which describe the behaviour of the velocity fluctuations. Here, problems of the same nature arise: if we define the instantaneous particle transport rate as $q_{s}=\gamma u_{p}$, where $\gamma$ is called the 'particle activity' (number of particles in motion per unit bed area) and $u_{p}$ is the particle velocity, then we can use the Reynolds decomposition and end up with $\bar{q}_{s}=\overline{\gamma u_{p}}+\overline{\gamma^{\prime} u_{p}^{\prime}}$. Experimental observations reveal the existence of large fluctuations in the particle activity: $\gamma^{\prime}=O(\bar{\gamma})$ (Cudden \& Hoey 2003; Ancey et al. 2008; Singh et al. 2009; Campagnol, Radice \& Ballio 2012). What is the consequence of these fluctuations at the macroscopic scale? May we discard them? Can we find algebraic closure equations? Or, on the contrary, must we develop non-algebraic closure equations relating $\gamma^{\prime}$ and $\bar{\gamma}$ ? If so, how are the macroscopic mass and momentum conservation equations affected?

It is difficult to find answers to these questions in the existing literature. A perusal of the literature would convince any reader that it is not for lack of trying. Over the last few decades, bed load transport has inspired a whole host of publications presenting different features of particle motion (e.g. interactions between the bed morphology and water stream, the conditions for incipient motion), leading to a substantial body of theoretical work (García 2007). However, no complete and unified theory of bed load transport has emerged from the different models developed so far, even though the current models often have the same continuum framework and share many assumptions and components. Some points, which may seem obvious at a first glance, raise a number of subtle questions or consistency problems. A typical example 


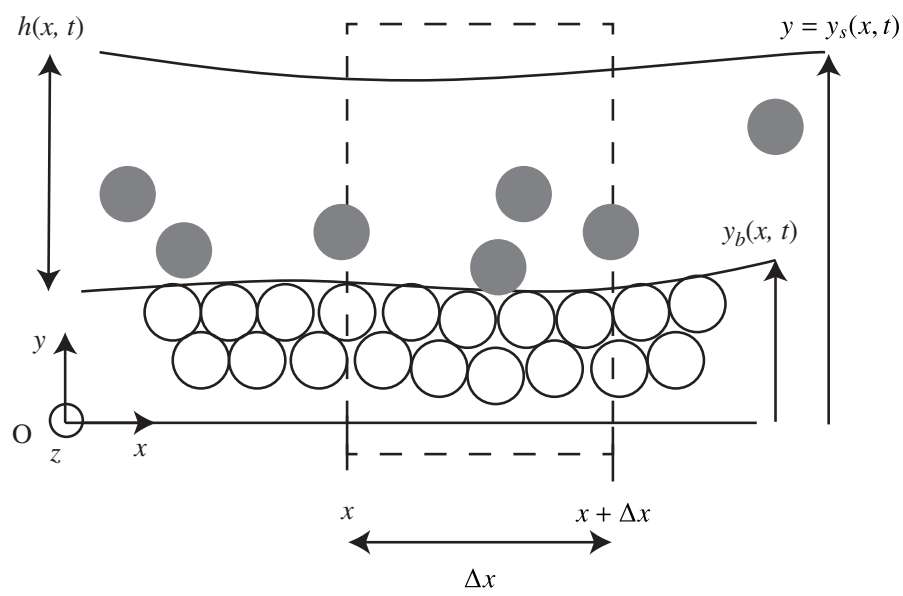

FIGURE 1. Schematic defining the flow configuration.

is the particle flow rate, which can be defined as a particle flux (Eulerian point of view) or in terms of particle jump lengths (Lagrangian point of view) (Ancey 2010; Furbish et al. 2012a; Ballio, Nikora \& Coleman 2013). It comes as no surprise that salient questions such as the relevance of fluctuations to the macroscopic behaviour of bed load transport have divided the community. An example is given by the first theoretical framework to include probabilistic calculations, developed by Einstein (1950), which roused the community's interest but also raised a number of criticisms and slipped into semiobscurity (Yalin 1972). The trouble caused by the nature of fluctuations is best understood by looking at their appearance in the macroscopic description of bed load transport.

For one-space variable problems, the simplest morphodynamic model we can envision comprises the shallow-water (Saint-Venant) equations for the conservation of mass and momentum of the water phase and the Exner equation for the continuity equation of the bed (see figure 1):

$$
\begin{aligned}
\frac{\partial h}{\partial t}+\frac{\partial h \bar{v}}{\partial x} & =0, \\
\frac{\partial h \bar{v}}{\partial t}+\frac{\partial h \bar{v}^{2}}{\partial x}+g h \cos \theta \frac{\partial h}{\partial x} & =g h \sin \theta-\frac{\tau_{b}}{\varrho}, \\
\left(1-\zeta_{b}\right) \frac{\partial y_{b}}{\partial t}=-\frac{\partial \bar{q}_{s}}{\partial x} & =D-E,
\end{aligned}
$$

in which $h(x, t)=y_{s}-y_{b}$ denotes the flow depth, $y_{b}(x, t)$ and $y_{s}(x, t)$ the positions of the bed and free surfaces, $\bar{v}$ the depth-averaged velocity, $x$ the downstream position, $t$ time and $\varrho$ the water density. Here $\tau_{b}$ is the bottom shear stress, $\zeta_{b}$ the bed porosity, $\bar{q}_{s}$ the average bed load transport rate and $D$ and $E$ represent the deposition and entrainment rates. The bed slope is defined as $\tan \theta=\partial_{x} y_{b}$. In most models based on (1.1)-(1.3), the governing equations are closed by empirical relationships for the flow resistance $\tau_{b}$ (Katul et al. 2002; Ferguson 2012) and the sediment transport rate $\bar{q}_{s}$ (Graf \& Altinakar 2005; García 2007), both being functions of the flow variables $\bar{v}$ and $h$, and additional parameters (e.g. bed roughness and slope).

Whereas the Saint-Venant equations are classic and their physics is seldom called into question, the coupling with the Exner equation leads to numerous difficulties both 
physically and mathematically (Parés 2006; Fasolato et al. 2011). Several derivations of the Exner equation have been proposed for different situations including variations in sediment properties (e.g. density, velocity, concentration, size fraction) or changes in the boundary conditions (e.g. tectonic uplift for landscape dynamics problems) (Defina 2000; Parker, Paola \& Leclair 2000; Paola \& Voller 2005; Lanzoni 2008; Ancey 2010; Furbish et al. 2012a). A central theme in all of these derivations is that the Exner model is an averaged equation that specifies the rate of buildup/erosion of the bed surface as a function of the sediment flux through the surface bounding the control volume over which the averaging has been done. The average sediment transport rate has non-trivial effects on the flow dynamics owing to the strong nonlinearities and coupling in the governing equations (1.1)-(1.3). Indeed, the sediment flux affects the bed surface $y_{b}(x, t)$ (thus its slope angle $\theta$ ) through the Exner equation (1.3), and it may also influence flow resistance depending on the empirical parametrization chosen for the bottom shear stress $\tau_{b}$ (Recking et al. 2008). While the coupling between sediment transport and water stream flow is usually seen as the primary agent of bed form development on various scales from small-scale patterns such as ripples to large-scale patterns such as dunes (Coleman \& Nikora 2009; Seminara 2010), linear stability analyses of the governing equations and numerical simulations do not show any instability in the governing equations (1.1)-(1.3) for Froude numbers lower than two (Balmforth \& Provenzale 2001; Charru 2011). This failure is often interpreted as a lack of physical details in the Saint-Venant approach, which prompts the use of models of greater complexity that provide a better physical picture of the turbulent flow and particle entrainment/transport (Seminara 2010). In particular, when coupling the Exner equation with a rotational two- or three-dimensional (non-depth-averaged) model of the water stream, linear stability analysis shows that one-dimensional (dunes) and two-dimensional (bars) bed forms are successfully reproduced (Colombini \& Stocchino 2011, 2012).

An alternative approach to pattern formation highlights the part played by random fluctuations of the particle transport rate $q_{s}$ in the development of bed forms. Analogies can be drawn with many nonlinear physical systems, in which fluctuations can produce spatially regular structures as a result of noise-induced transitions between different states of the system (Sagués, Sancho \& García-Ojalvo 2007). In the absence of a more fundamental understanding of bed load transport fluctuations, the simplest idea has been to add a noise term to the governing equations. In doing so, Jerolmack \& Mohrig (2005) showed that the growth and steady-state dimensions of sand dunes can be successfully captured using white noise in the Exner equation (1.3). More recently, making use of statistical mechanics arguments, Furbish et al. (2012a) have demonstrated that the macroscopic Exner equation can be related to the details of particle trajectories as long as the fluctuating particle velocities are properly accounted for in the definition of the bed load sediment flux. These authors have shown that within this framework, the Exner equation includes diffusive terms (second-order space derivative).

We begin by presenting the physical system studied and the notation used throughout the body of this paper (see $\S 2$ ) and the supplementary material, available at http://dx.doi.org/10.1017/jfm.2014.074. In $\S 3$, we focus our attention on the particle flux through a control volume. We generalize the Markov process model presented in an earlier publication (Ancey et al. 2008). The novelty lies in the use of the Poisson representation, which is an elegant and exact technique that makes it possible to use continuous probability distributions instead of the original discrete distributions without any simplifying approximations. The mapping between discrete 
and continuous distribution spaces allows us to generalize the model developed for an isolated volume to an array of adjacent volumes (see $\S 4$ ). A continuum formulation is then derived and compared with existing forms of the Exner equation. Finally, some experimental data drawn from our previous experimental campaigns are used to provide the proof of concept (see $\S 5$ ).

\section{Physical problem}

\subsection{Motivation}

The one-dimensional Saint-Venant-Exner equations (1.1)-(1.3) have been used in a variety of settings covering a wide range of length and time scales. Typical examples include the simulation of outburst flows with intense sediment transport (Bohorquez \& Darby 2008), the sediment budget together with the bed evolution over a few decades (Ferguson \& Church 2009), the long-term evolution of rivers at the reach scale (Parker \& Izumi 2000) or the watershed scale (Fowler, Kopteva \& Oakley 2007). In most cases, comparison with field data reveals qualitative agreement but also significant errors often up to one or several orders of magnitude in the quantification of the sediment budget and bed evolution, which may undermine the adequacy of the onedimensional Saint-Venant-Exner equations (1.1)-(1.3).

Several arguments have been put forward to explain the shortcomings of this approach. For meso-timescales (typically within the 10-10 4 years range), geomorphologists argue that river bed dynamics are dominated by randomness as a result of climatic and tectonic forcings, which cause the river to change course and create new channels on a random basis (a phenomenon called avulsion) (Paola 2000; Tipper 2007). On shorter timescales (shorter than 10 years), scientists blame nonlinearity as the main source of error. Indeed as all of the flow variables are averaged over the channel width, the details of the actual stress distribution and the velocity field across the flow section (including secondary currents and acceleration/deceleration due to curvature effects) are ignored, which biases the calculation of the transport rates (Ferguson \& Church 2009; Recking 2013). While the one-dimensional Saint-Venant-Exner equations (1.1)-(1.3) can be used to compute the bed profile evolution, they do not admit important spatial structures such as bars and other planform patterns (Church 2006; Bohorquez \& Darby 2008; Colombini \& Stocchino 2011). The role played by the grain size distribution has also been pointed out as a key factor in obtaining more realistic predictions (Ferguson \& Church 2009).

More recently, the role played by the fluctuations of the sediment transport rate has been highlighted as another source of trouble in the numerical simulations of sediment transport. While the existence of these fluctuations has long been recognized from field measurements (Cudden \& Hoey 2003; Bunte \& Abt 2005), they have been mostly interpreted as a result of bed form migration (Gomez 1991) rather than an intrinsic process. From this perspective, their effects should be determined when solving (1.1)-(1.3) numerically and taking bed form development and migration into account. Yet, Recking et al. (2012) have shown that the bed load equation used in (1.3) should be carefully chosen as a function of the timescale of the problem. By testing different bed load transport models against instantaneous field measurements, volume accumulated at the event scale, volume accumulated at the annual and interannual scales, and time-integrated flume measurements, these authors found that at very short timescales (say, a few minutes), the bed load equations failed to predict the mean sediment transport rate to within one order of magnitude. The results were especially poor for coarse-bed rivers and steep slopes. In contrast, on much longer 
timescales (say, one year), the predictions more closely matched the field data, with relative errors (computed/measured volume of sediment) lower than five in most cases.

To us, apart from the usual sources of complexity cited in the literature (hiding effects, turbulence and the threshold of incipient motion, flow seepage, bed topography, etc.), the poor performance of bed load equations on short timescales is a clear indication that the fluctuations of the sediment transport rate are not only very large, but they also affect the macroscopic behaviour. Laboratory experiments reveal that even under strictly steady-state conditions and well-sorted particles, sediment transport rates exhibit considerable noise (Böhm et al. 2004; Ancey et al. 2006; Singh et al. 2009; Radice 2009; Campagnol et al. 2012).

\subsection{Problem addressed}

Our guess is that, if fluctuations are important in the macroscopic description of bed load transport, then the averaged Exner equation (1.3) will not be able to properly account for the bed evolution or particle flux, or at least, this equation will be a gross approximation of reality. It will certainly hold true on average, but each experimental or field measurements being particular realizations, empirical averages may depart significantly from the theoretical average.

To clarify this point, we need to take a closer look at the microdynamics of the bed evolution as a result of entrainment and deposition of particles. The microstructural analysis underpinning the derivation of the Exner equation is known to be a daunting problem (Paola \& Voller 2005; Vollmer \& Kleinhans 2007; Lanzoni 2008; Coleman \& Nikora 2009). The only way to make progress is by simplifying the picture of the real system by keeping only the salient features.

Here, we develop an analytical model of bed load transport, that is, the transport of particles by rolling, sliding or saltation in a turbulent water stream. Particles move mostly in contact with the bed surface, but may experience brief periods in the stream. This mode of transport contrasts with suspended load, in which fine particles are maintained in suspension by turbulence. The main objective is to calculate the particle transport rate from a microstructural analysis of particle motion within a control volume (Eulerian description). Figure 1 shows the flow configuration. To achieve analytical results, we need to introduce a number of simplifications.

(i) The sediment comprises spherical particles of equal diameter $d$ and density $\varrho_{p}$.

(ii) We consider a two-dimensional steady water stream flowing down a sloping bed composed of particles identical to those transported. The bed breadth $B$ is assumed to be indefinitely large.

(iii) The water flow is characterized by its depth-averaged velocity $\bar{v}(x, t)$ and flow depth $h(x, t)$, which are assumed to be prescribed and independent of the sediment transport. The water flow is turbulent, but the details of the turbulence and velocity field are ignored. Turbulence dissipation and flow resistance due to the particles are entirely encoded in the $\tau_{b}(\bar{v}, h)$ expression, which will not be studied here.

(iv) The concentration of moving particles is small and so particle interactions may be neglected. In terms of the bed load transport regime, this also means that the bed shear stress narrowly exceeds the threshold for incipient sediment motion.

(v) The water stream drives the sediment phase: the particle phase is subordinate to the water phase in that the mean particle velocity $\bar{u}_{s}$ is controlled by the water flow conditions, but due to particle exchanges (entrainment/deposition) between 
the bed and stream as well as particle velocity fluctuations, the instantaneous particle flux $q_{s}$ undergoes variations of different magnitudes.

(vi) The bed is initially flat and here we do not consider the development of bed forms, even though after a finite time such bed forms are likely to develop and affect water flow and sediment transport.

(vii) We do not discriminate between rolling and saltation and treat both motions as defining a single species which we call the moving particles. We assume that the number of particles making up the bed is infinite, i.e. whenever a particle at the bed interface is set in motion, the shape of the interface is altered, but not the number of particles available to entrainment at the bed interface.

(viii) As we study steady uniform flows over flat beds (free of patterns), we do not address the dependence of the model coefficients on the Shields stress or any other parameterization of the flow conditions. These coefficients (e.g. entrainment and deposition rate coefficient) are thus constant in the following developments. Note that the final structure of the governing equations will not be affected by this assumption.

Our theoretical developments have been guided by similar problems that arise in chemical kinetics and population dynamics, from which we have borrowed a number of mathematical tools, in particular the concepts of Markov processes, continuous-time random walk and master equation (Gardiner 1983; Gillespie 1992), the Poisson representation (Gardiner \& Chaturvedi 1977) and the Langevin equation (Gardiner 1983). This paper also generalizes our first attempt to deal with a stochastic treatment of entrainment and deposition of coarse particles (Ancey et al. 2008). Although these mathematical tools are well-established, they are not necessarily widespread, in particular within the sediment transport community. For this reason, much of the mathematical detail has been left out of this paper and placed in the supplementary material. The model developed here aims to show the connections between the microand macro-scales for the particle flux. As for any idealized formulation, a tradeoff between physical scope and mathematical tractability has had to be found.

\subsection{Notation and definitions}

Different definitions can be used for the sediment flux (Ancey 2010; Furbish et al. 2012a; Ballio et al. 2013). While a definition based on ensemble averaging fits in perfectly with our objective of describing the sediment flux fluctuations, it presents many theoretical and practical difficulties, which push us to use a volume-averaged definition, as explained in the supplementary material. We therefore define the sediment transport rate per unit width as a volume average of the particle velocities at the microscopic scale over a control volume $\mathscr{V}$ of side $\Delta x$

$$
q_{s}(x, t ; \mathscr{V} ; \bar{v}, h)=N \frac{\varpi_{p}}{\Delta x} \mathscr{U}_{n}=\gamma \mathscr{U}_{n}=\frac{\varpi_{p}}{\Delta x} \sum_{i=1}^{N} u_{p, i},
$$

where, following Furbish et al. (2012a), we refer to $\gamma=N \varpi_{p} / \Delta x$ as the "particle activity' (i.e. the volume of moving particles per unit bed area), $\varpi_{p}=4 \pi d^{3} /(24 B)$ is the particle volume per unit width, $N$ represents the number of particles whose centres of mass lie within $\mathscr{V}, u_{p, i}$ denotes the streamwise component of the motion of the centre of mass of particle $i$, and $\mathscr{U}_{n}=\sum_{i=1}^{N} u_{p, i} / N$ is the arithmetic average of these 
velocities. Equally, we can define the sediment transport rate as the number of moving particles per unit time

$$
\dot{n}=\frac{N}{\Delta x} \mathscr{U}_{n} .
$$

The main difference between these two definitions is that $\gamma$ (or $q_{s}$ ) is a continuous random variable, whereas $N$ is discrete, which gives different possibilities for analysis. The error made in considering an equivalence between $\gamma$ and $N$ is vanishingly small provided that $\gamma$ is locally non-zero (see the supplementary material).

The notation used in this paper is as standard as possible. Greek letters generally denote parameters, e.g. entrainment and deposition coefficients in our model. Roman letters are used for random variables and the particular value they can take is denoted by lowercase letters. For instance, $N$ is a random variable describing the number of moving particles; the probability that $N$ takes the value $n$ is denoted by $P_{n}(n)=$ $\operatorname{Prob}(N=n)$. Angular brackets denote an ensemble average.

To make the notation less cluttered, we do not follow this rule strictly. This is the case for $\dot{n}$, for which we keep the same notation for the variable and its random value. The average or expectation of a distribution is indicated using angle brackets, e.g. $\langle N\rangle=\sum_{n} P_{n}(n) n$ is the mean number of particles. The variance is denoted var. Here probability functions $P$ are probability density functions (or probability mass functions for discrete variables). Wherever necessary, a subscript indicates to which random variable a particular density function refers. The only exception is the subscript $s$, which pertains to steady flow conditions or processes. An index of variables can be found in the supplementary material.

\section{Stochastic behaviour for a fixed control volume}

There is accumulating evidence that much of the fluctuations in $q_{s}$ come from the variation in $N$ (Ancey et al. 2008; Radice, Ballio \& Nikora 2009; Roseberry, Schmeeckle \& Furbish 2012), at least at fairly low water flow rates when the sediment flux is not intense. If so, the crux of the issue is then the proper determination of $N$ or the 'activity' $\gamma$. We do not gloss over the importance of particle velocity, however, its calculation is more easily amenable to analysis when we focus on short timescales. On longer timescales, when particle paths are characterized by alternating mobile and resting phases, this analysis is more involved (Ganti et al. 2010; Zhang et al. 2012). In the former case, the central limit theorem applies and we expect that the arithmetic mean $\mathscr{U}_{n}$ tends to a well-defined mean particle velocity $\bar{u}_{s}$. There are a number of models available for calculating the mean velocity of a single particle as a function of the flow velocity or shear stress (Wiberg \& Smith 1985; Ancey et al. 2002; Niño et al. 2002; Chatanantavet et al. 2013), as well as its statistical properties (Lajeunesse, Malverti \& Charru 2010; Furbish, Roseberry \& Schmeeckle 2012b). Therefore, for the sake of simplicity, we assume that the mean particle velocity $\bar{u}_{s}(x, t ; \bar{v}, h)$ can be determined independently and is fully controlled by the water stream.

In this section, we consider the time variation in the number of moving particles within a fixed control volume. We first summarize our earlier results of the calculation of the number of moving particles within this control volume ( $\$ 3.1)$. Compared with our former developments (Ancey et al. 2008), we focus here on the exchanges between the bed and stream without paying attention to the advection of particles. We also highlight that the final probability distribution of $N$ can be regarded as a generalized Poisson distribution, a result that will be crucial to the derivation of a 


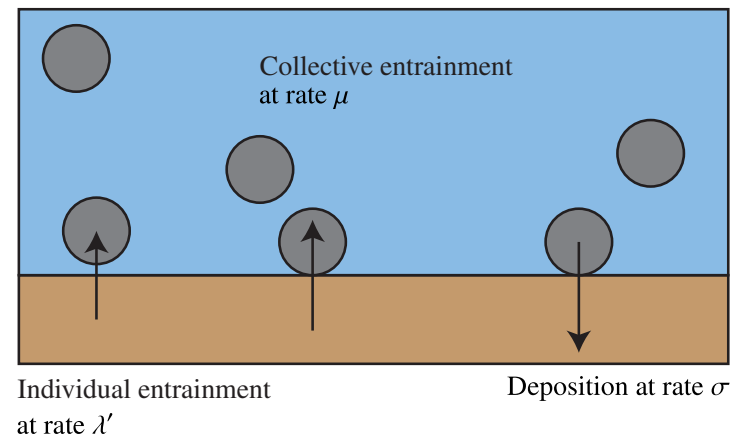

FIGURE 2. (Colour online) The number of moving particles observed within the window varies with time depending on the number of particles being entrained from/deposited on the stationary bed.

continuum model. Then, we introduce a simple model to compute the probability distribution of the particle velocity $(\S 3.2)$. All of these results will be reused when generalizing the model to an array of adjacent cells in the next section. As we skip the mathematical details, appendix A provides further information on the mathematical techniques used here, while appendix $\mathrm{B}$ shows how the particle flow rate and its probability distribution can be computed from the distributions of $N$ and $u_{p}$ using our definition (2.1).

\subsection{Number of moving particles}

We start our analysis by studying the evolution of $N$ moving particles within a given control volume $\mathscr{V}$. The number of particles may vary with time as a result of various events such as deposition and entrainment (see figure 2). A convenient framework for the investigation of the statistics of these exchanges is the theory of birth-death Markov processes, widely used in population-dynamics models or chemical kinetics (Gillespie 1992).

If there are $N$ moving particles within the control window, the probability of deposition within the time increment $\delta t$ is $\sigma N \delta t$, with $\sigma$ the deposition rate. For entrainment, we assume that there are two processes referred to as individual and collective entrainment resulting in a probability of entrainment $P=\left(\lambda^{\prime}+\mu N\right) \delta t$, where $\lambda^{\prime}$ and $\mu$ denote the individual and collective entrainment rates, respectively. Collective entrainment acts as a feedback loop: as will be shown later, $\mu$ is a key parameter, which controls the development and strength of wide fluctuations. A caveat is in order: here, collective entrainment implies that the probability of entrainment depends not only on the flow conditions (through $\lambda^{\prime}$ ), but also on the number of moving particles (through $\mu$ ) as these can impact the bed and impart momentum to the bed particles, favouring their entrainment. In contrast with the physics of phase transition, it does not involve the existence of long-range correlations. It does not mean that there are massive departures of particles (avalanches) within short time spans. For subsequent use, we also introduce a volumetric particle entrainment rate per unit length $\lambda=\lambda^{\prime} \varpi_{p} / \Delta x$ and the differential rate $\kappa=\sigma-\mu$ between deposition and collective entrainment. 
Using discrete Markov process theory, we can show that the probability of finding $N=n$ particles at time $t$ is given by the forward master equation

$$
\frac{\partial}{\partial t} P(n, t)=(n+1) \sigma P(n+1, t)+\left(\lambda^{\prime}+(n-1) \mu\right) P(n-1, t)-\left(\lambda^{\prime}+n(\sigma+\mu)\right) P(n, t) .
$$

When the collective entrainment $\mu$ is non-zero, the steady-state solution to (3.1) is the negative binomial distribution

$$
P_{s}(n)=\operatorname{NegBin}\left(n ; r_{n b}, p\right)=\frac{\Gamma(r+n)}{\Gamma\left(r_{n b}\right) n !} p^{r_{n b}}(1-p)^{n}, \quad n=0,1, \ldots,
$$

with $r_{n b}=\lambda^{\prime} / \mu$ and $p=1-\mu / \sigma$, and where $\Gamma$ denotes the gamma function. The mean is

$$
\langle N\rangle=\frac{\lambda^{\prime}}{\sigma-\mu},
$$

and the variance is

$$
\operatorname{var} N=\frac{\lambda^{\prime} \sigma}{(\sigma-\mu)^{2}}
$$

For $\mu=0$, we obtain the Poisson distribution of rate $r_{p}=\lambda^{\prime} / \sigma$,

$$
P_{s}(n)=\frac{\left(r_{p}\right)^{n}}{n !} \mathrm{e}^{-r_{p}}, \quad n=0,1, \ldots
$$

It is worth noting that despite appearances, the solutions corresponding to the $\mu>$ 0 and $\mu=0$ cases are connected. Indeed, the negative binomial distribution can be interpreted as a compound probability distribution where the mixing distribution of the Poisson rate is a gamma distribution

$$
P_{s}(n)=\int_{a} \frac{\mathrm{e}^{-a} a^{n}}{n !} G a(a ; \alpha, \beta) \mathrm{d} a=\operatorname{NegBin}\left(n, r_{n b}, p\right)=\frac{\Gamma\left[r_{n b}+n\right]}{\Gamma\left[r_{n b}\right]} p^{r_{n b}}(1-p)^{n},
$$

with $\alpha=r_{n b}=\lambda^{\prime} / \mu$ and $\beta=1 / p-1=\mu /(\sigma-\mu)$. So, interestingly, we retrieve the Poisson distribution even in the case $\mu>0$, but hidden behind the stochastic variations of its rate $r_{p}$.

A physical interpretation can then be proposed. In the absence of collective entrainment $(\mu=0)$, the behaviour is Poissonian with a fixed rate $r_{p}=\lambda^{\prime} / \sigma$ dictated by entrainment and deposition of individual particles. When collective entrainment occurs $(\mu>0)$, the behaviour may still be seen as Poissonian, but with a random rate. This random rate follows a gamma distribution, whose parameters are greatly influenced by $\mu$, especially when $\mu \rightarrow \sigma(\beta \rightarrow \infty)$. This observation prompts us to seek a generalized solution to the forward master (3.1) in the form of a Poisson representation (see appendix A). In the following, this feature will be exploited to derive a continuum description of the $N$ variations in an array of adjacent cells. 


\subsection{Particle velocity fluctuations}

After considering the kinetics of exchanges between the stream and the bed, let us take a closer look at the part played by particle velocity fluctuations in the sediment transport rate. We assume that the motion of a given particle, which was initially at a position $\boldsymbol{x}_{0}$ at $t=0$, can be described using the following Langevin equation

$$
\begin{aligned}
\frac{\mathrm{d} X}{\mathrm{~d} t} & =U, \\
t_{r} \frac{\mathrm{d} U}{\mathrm{~d} t} & =-\left(U-\bar{u}_{s}\right)+\sqrt{2 D_{u}} \xi(t),
\end{aligned}
$$

a where $X$ is the abscissa of the centre of mass, $U$ denotes its velocity, $t_{r}$ is a relaxation time, $D_{u}$ is the equivalent of a particle diffusivity, $\xi(t)$ is a white noise term. In essence, equations (3.7) and (3.8) state that the particle acceleration comprises a deterministic component and a stochastic term that reflects the random fluctuations induced by the water stream. On average and in the long run $t \gg t_{r}$, the particle velocity tends to the mean sediment velocity $\bar{u}_{s}(\bar{v}, h)$; if we do not discard the fluctuations, a crude approximation of the particle velocity is then $U=\bar{u}_{s}+\sqrt{2 D_{u}} \xi(t)$. Stochastic equations (3.7) and (3.8) are equivalent to the Fokker-Planck equation for the (Lagrangian) distribution function $\hat{P}(x, u, t)$

$$
\frac{\partial \hat{P}}{\partial t}=-\frac{\partial}{\partial x}(u \hat{P})+\frac{\partial}{\partial u}\left(\hat{P} \frac{u-\bar{u}_{s}}{t_{r}}\right)+\frac{1}{t_{r}^{2}} \frac{\partial^{2}}{\partial u^{2}}\left(D_{u} \hat{P}\right) .
$$

Next, to derive the velocity distribution for a system at equilibrium, we assume that there is no dependence of $P_{u}$ and of auxiliary variables $\left(u_{s}(\bar{v}, h)\right.$ and $\left.D_{u}(\bar{v}, h)\right)$ on $x$ and $t$. The equilibrium velocity distribution $P_{u}^{e q}$ can then be derived straightforwardly from the Fokker-Planck equation (3.9)

$$
P_{u}^{e q}(u)=\sqrt{\frac{2 t_{r}}{\pi D_{u}}} \frac{1}{1+\operatorname{erf}\left(\bar{u}_{s} \sqrt{t_{r}} / \sqrt{2 D_{u}}\right)} \exp \left(-\frac{t_{r}\left(u-\bar{u}_{s}\right)^{2}}{2 D_{u}}\right),
$$

which is a truncated Gaussian distribution. Therefore, $\bar{u}_{s}$ is still the most likely velocity, but it is not the mean value. The mean velocity at equilibrium $\langle u\rangle_{e q}$. is somewhat higher than the mean velocity of a single particle $\bar{u}_{s}$

$$
\langle u\rangle_{e q .}=\bar{u}_{s} F_{e q .}(\zeta)
$$

with

$$
F_{e q .}(\zeta)=1+\frac{1}{\zeta} \sqrt{\frac{2}{\pi}} \frac{\exp \left(-\zeta^{2 / 2}\right)}{1+\operatorname{erf}(\zeta / \sqrt{2})},
$$

with $\zeta=\bar{u}_{s} / \sqrt{D_{u} / t_{r}}$, but as shown by figure 3 , provided $\zeta>1$, we have $\langle u\rangle_{e q} \approx \bar{u}_{s}$. Its variance var $u$ decreases rapidly as $\zeta^{-2}$, which means that it is nearly constant (and proportional to $\left.D_{u} / t_{r}\right)$ :

$$
\operatorname{var} u=\bar{u}_{s}^{2}\left(1+\frac{1}{\zeta^{2}}+\frac{1}{\zeta} \sqrt{\frac{2}{\pi}} \frac{\exp \left(-\zeta^{2 / 2}\right)}{1+\operatorname{erf}(\zeta / \sqrt{2})}\right)-\langle u\rangle_{e q .}^{2} \propto 0.38 \bar{u}_{s}^{2} \zeta^{-2}=0.38 \frac{D_{u}}{t_{r}}
$$




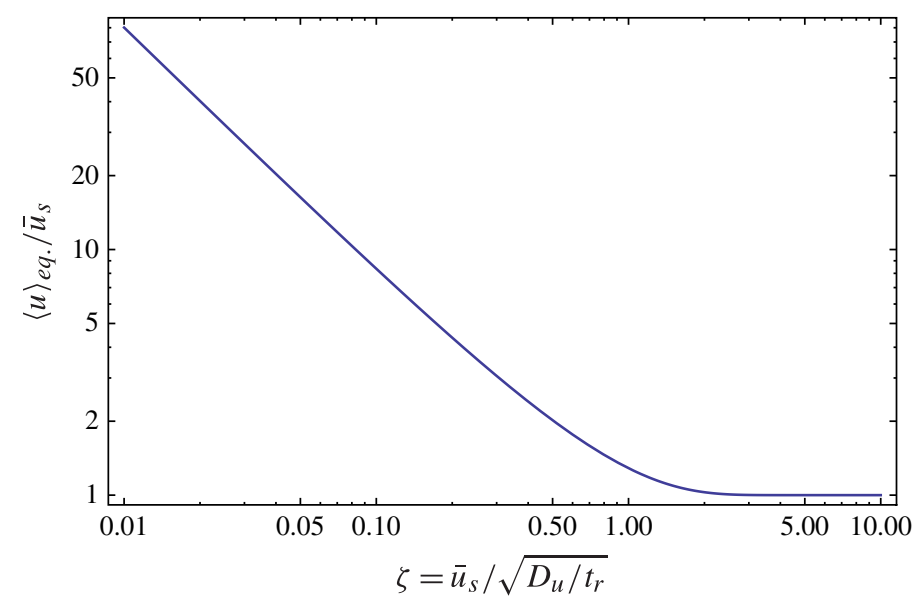

FIGURE 3. (Colour online) Variation of the ratio $\langle u\rangle_{e q .} / \bar{u}_{s}$ as a function of $\zeta$.

\section{Generalization to an array of adjacent cells}

We are now poised to generalize the results to a collection of cells by considering particle transport, entrainment and deposition. The last two mechanisms have been studied at length in the previous section. As they are local processes, they will not cause problems when generalizing the results from an isolated control volume to an array of adjacent cells. In contrast, particle transport requires more work. We will show that under certain constraints, particle transport can be broken into advection and (linear) diffusion. Furthermore, working with discrete random variables does not facilitate our analysis as the corresponding governing equation (3.1) does not lead to averaged equations that are compatible with the usual continuum mechanics equations. Here, we will take advantage of the remark made in $\S 3.1$ about the solution to (3.1), which can be interpreted as a generalized Poisson distribution, i.e. a Poisson distribution whose parameter is a continuous random number. The idea is to use the Poisson representation, which involves expanding any probability distribution into a sum of Poisson distributions. This representation is a Laplace-like transform, which allows us to pass from a discrete probability space (referred to as the $n$-space) to a continuous probability space (the $a$-space).

\subsection{Mean behaviour}

Let us start by computing the number of moving particles within $\mathscr{V}$ around $x$ :

$$
N(t ; \mathscr{V})=\int_{\mathscr{V}} \mathrm{d} x P_{x}(x, t),
$$

where $P_{x}(x, t) \mathrm{d} x$ is the probability of finding the centre of mass $\boldsymbol{x}=(x, y)$ of a moving particle within a vertical slice of fluid $[x, x+\mathrm{d} x]$. This probability $P_{x}(x, t)$ is the marginal probability density function of the Eulerian probability $P(x, u, t)$ : $P_{x}(x, t)=\int_{u} P(x, u, t) \mathrm{d} u$. Making use of the equivalence relation between Lagrangian and Eulerian probability distributions $P(x, u, t)=\int_{\mathscr{V}} \hat{P}\left(x^{\prime}, u, t\right) \delta\left(x^{\prime}-x\right) \mathrm{d} x^{\prime}$ (Minier \& Peirano 2001), we find that the variations of $P(x, u, t)$ are also given by the Fokker-Planck equation (3.9). Ideally, we would like to derive a governing equation 
for $P_{x}(x, t)$ from (3.9). Here we take advantage of the fact that equations (3.7) and (3.8) are identical to those used for the Brownian motion of particles placed in a potential: indeed, the water velocity $\bar{v}$ is seen as the equivalent of an external potential acting on the particles. We can then use the adiabatic-elimination technique to transform the Fokker-Planck equation (3.9) into a reduced Fokker-Planck equation for the Lagrangian distribution $\hat{P}_{x}(x, t)$ (Gardiner 1983)

$$
\frac{\partial \hat{P}_{x}}{\partial t}=-\frac{\partial}{\partial x}\left(\bar{u}_{s} \hat{P}_{x}\right)+\frac{\partial^{2}}{\partial x^{2}}\left(D_{u} \hat{P}_{x}\right) .
$$

We can take the ensemble average of (4.2) to obtain

$$
\frac{\partial}{\partial t}\langle\gamma\rangle+\frac{\partial}{\partial x}\left(\bar{u}_{s}\langle\gamma\rangle\right)=\frac{\partial^{2}}{\partial x^{2}}\left(D_{u}\langle\gamma\rangle\right)
$$

with $\langle\gamma(x, t)\rangle=\varpi_{p}\langle N(t ; \mathscr{V}) / \Delta x\rangle$ the average particle activity.

The last building block is to include bed exchanges. Owing to the linearity of the processes, we may simply add the mean variations resulting from bed exchanges as a source term in (4.3). We eventually end up with the following governing equation for the average particle activity

$$
\frac{\partial}{\partial t}\langle\gamma(x, t)\rangle+\frac{\partial}{\partial x}\left(\bar{u}_{s}\langle\gamma(x, t)\rangle\right)=\frac{\partial^{2}}{\partial x^{2}}\left(D_{u}\langle\gamma(x, t)\rangle\right)+\lambda-\kappa\langle\gamma(x, t)\rangle,
$$

with $\kappa=\sigma-\mu$ (the source term pertaining to bed exchanges is given by (A 36)). This is a linear advection diffusion equation with a source term. Albeit of very common structure, this equation yields many interesting insights into the physics of sediment transport. We will examine two features.

First, at this level of development, it is interesting to compare our microstructural description of sediment transport with other approaches including continuum models and probabilistic formulations of the bed load sediment flux derived from Lagrangian analysis of particle motion (Furbish et al. 2012a). The first step is to note that (4.4) can also be cast in the following form

$$
\frac{\partial}{\partial x} \mathscr{Q}(x, t)=E(x, t)-D(x, t)-\frac{\partial}{\partial t}\langle\gamma\rangle,
$$

with $\mathscr{Q}=\bar{u}_{s}\langle\gamma\rangle-\partial_{x}\left(D_{u}\langle\gamma\rangle\right), E=\lambda+\mu\langle\gamma\rangle$ and $D=\sigma\langle\gamma\rangle$. Interestingly, if we borrow the definition of the sediment flux rate from Furbish et al. (2012a) and refer to $\mathscr{Q}$ as the macroscopic sediment transport rate, then (4.5) is the generalized Exner equation established by a number of authors (Parker et al. 2000; Cao, Day \& Egashira 2002; Paola \& Voller 2005). Note that the standard equation (1.3) does not usually include the time variation in the particle activity $\partial_{t}\langle\gamma\rangle$ as this term is vanishingly small. Indeed, using dimensional analysis, Cui et al. (2005) showed that provided that the ratio $\epsilon=\bar{q}_{s} / q_{w}$ (with $q_{w}$ the water discharge) remains small, the time variation $\partial_{t}\langle\gamma\rangle$ is second order. Thus to leading order, the bed evolution $\partial_{t} y_{b}$ is controlled by the gradient $\partial_{x} \bar{q}_{s}$.

Second, we note that, owing to the diffusive term $\partial_{x x}\left(D_{u}\langle\gamma\rangle\right)$, the governing equation (4.4) for $\langle\gamma\rangle$ differs from pure advection models used in most morphodynamic models (Jerolmack \& Mohrig 2005; Lajeunesse et al. 2010). This is, however, not contradictory as many advection-driven processes are the end members of 
advective-diffusive processes. The usual argument is to introduce a Péclet number to delineate the flow regimes. Equation (4.4) arises in a variety of contexts including alloy solidification (Fowler 1997), traffic jams (Helbing 2001), reaction-diffusion systems (Méndez, Fedotov \& Horsthemke 2010) and transport of contaminants and solutes through porous media (Logan 2001), and a similar form was also proposed to model the dispersion of sediment pulses in gravel-bed rivers (Lisle et al. 2001). Numerous exact solutions, including similarity and travelling wave solutions, are known for specific boundary initial value problems. Here we focus on particle propagation from a concentrated instantaneous source. Let us assume that initially, the flow is at equilibrium, with the particle activity given by (4.6) and constant mean sediment velocity $\bar{u}_{s}$, and we disrupt the balance between entrainment and deposition by adding an instantaneous source of sediment located at $x=0$. We then study the evolution of the perturbation term $\left\langle\gamma^{*}\right\rangle=\langle\gamma\rangle-\langle\gamma\rangle_{s}$ subject to the initial condition $\left\langle\gamma^{*}\right\rangle=\Gamma_{0} \delta(x)$, where $\Gamma_{0}$ denotes the strength of the source (i.e. the volume of sediment released) and $\langle\gamma\rangle_{s}$ is the steady-state solution

$$
\langle\gamma\rangle_{s}=\frac{\lambda}{\sigma-\mu} .
$$

In a dimensionless form, equation (4.4) reads

$$
P e\left(\frac{\partial}{\partial \tilde{t}}\left\langle\tilde{\gamma}^{*}\right\rangle+\frac{\partial}{\partial \tilde{x}}\left\langle\tilde{\gamma}^{*}\right\rangle\right)=\frac{\partial^{2}}{\partial \tilde{x}^{2}}\left\langle\tilde{\gamma}^{*}\right\rangle-R^{2}\left\langle\tilde{\gamma}^{*}\right\rangle,
$$

where we have introduced the scaled variables $x=\tilde{x} \Delta x, t=\tilde{t}\left(\Delta x / \bar{u}_{s}\right)$ and $\gamma^{*}=$ $\tilde{\gamma}^{*}\left(D_{u}^{2} / \Delta x\right)$. We define the Péclet number as the ratio of the time $t_{d}=\Delta x^{2} / D_{u}$ necessary for a diffusing particle to travel a characteristic length $\Delta x$ to the time for a particle to be advected by the stream $t_{a}=\Delta x / \bar{u}_{s}$ :

$$
P e=\frac{t_{d}}{t_{a}}=\frac{\bar{u}_{s} \Delta x}{D_{u}} .
$$

We also introduce the correlation length $\ell_{c}=\sqrt{D_{u} / \kappa}$ associated with the diffusionreaction equation (see $\S 4.3$ for an interpretation) and the ratio $R=\Delta x / \ell_{c}$. In the limit $P e \rightarrow \infty$, equation (4.7) degenerates into a purely hyperbolic equation with a sink term. The method of characteristics leads to a solution of the form

$$
\left\langle\tilde{\gamma}^{*}\right\rangle=\tilde{\Gamma}_{0} \delta(\tilde{x}-\tilde{t}) \exp \left(-\frac{R^{2}}{P e} \tilde{t}\right),
$$

which shows that the initial pulse is transported along the characteristics $\tilde{x}=\tilde{t}$ (thus at constant velocity) by the stream with no deformation, but its strength is attenuated exponentially over time. For finite values of the Péclet number, making use of Fourier transformation in the space variable (see the supplementary material) leads to the solution

$$
\left\langle\tilde{\gamma}^{*}\right\rangle=\frac{\tilde{\Gamma}_{0} \sqrt{P e}}{\sqrt{4 \pi \tilde{t}}} \exp \left[-P e\left(\frac{(\tilde{x}-\tilde{t})^{2}}{4 \tilde{t}}+\left(\frac{R}{P e}\right)^{2} \tilde{t}\right)\right] .
$$

In contrast to homogeneous advection diffusion problems, the existence of a sink term implies that the volume of sediment released is not conserved, but decreases 

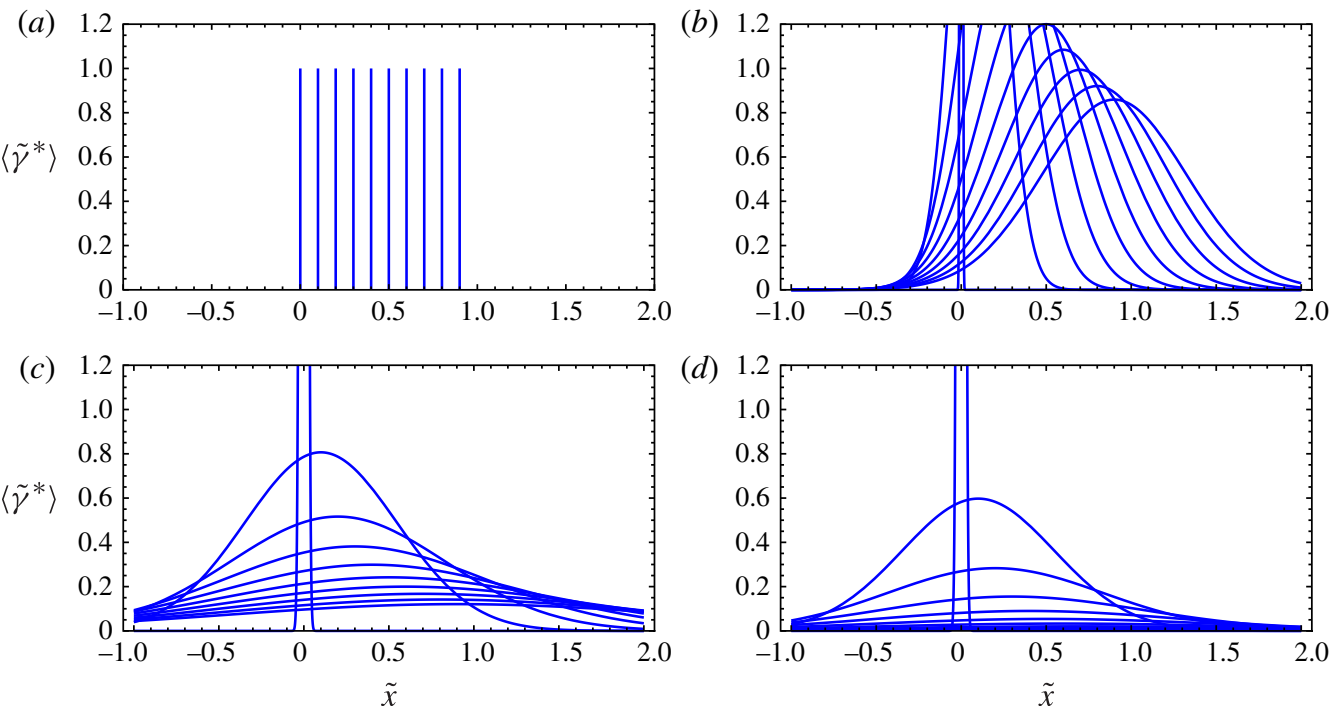

FIGURE 4. (Colour online) Evolution of the perturbation $\left\langle\tilde{\gamma}^{*}\right\rangle$ for $\tilde{t}=0$ to 10 by step of size unity: (a) $P e=10^{5}$ and $R=1$; (b) $P e=10$ and $R=1 ;(c) P e=1$ and $R=1$; and (d) $P e=1$ and $R=2$.

exponentially over time (bed aggradation): $\tilde{V}=\int\left\langle\tilde{\gamma}^{*}\right\rangle \mathrm{d} \tilde{x}=\tilde{\Gamma}_{0} \exp \left[-R^{2} \tilde{t} / P e\right]$. Equation (4.10) shows that at short times in the neighbourhood of the source at $\tilde{x}=0$, the solution $\left\langle\tilde{\gamma}^{*}\right\rangle$ decreases as $\tilde{t}^{-1 / 2} \mathrm{e}^{-\tilde{t}}$, but at long times, the decay rate is much more pronounced: $\left\langle\tilde{\gamma}^{*}\right\rangle \propto \tilde{t}^{-1 / 2} \mathrm{e}^{-\tilde{t}^{2}}$. The transition time is approximately $\tilde{t} \sim 4(R / P e)^{2}$. In that case the solution comprises advection of the initial cloud of particles, diffusion and reduction of the volume. Note that the limiting case $P e \rightarrow 0$ has no physical solution and for $P e>0$, the initial source of sediment spreads at infinite velocity as is the case for any linear diffusion problem. Solutions (4.9) and (4.10) show that a wealth of behaviour can be observed in a window of length $\Delta x$ depending on the dimensionless groups $P e$ and $R$ (or in terms of the physical parameters, $D_{u}, \bar{u}_{s}$ and $\kappa)$. This also shows the importance of the collective entrainment parameter $\mu$ since, at least formally, this parameter can come very close to the deposition rate $\sigma$, leading to large values of the correlation length $\ell_{c}$, thus relatively small values of the ratio $R$. In this case (for $R \ll P e^{-1 / 2}$ ), the perturbation propagates with little loss of mass whereas in other cases, there is an exponential decay to the initial state.

Figure 4 shows the range of behaviour that can be observed depending on the actual values of $P e$ and $R$. Figure 4(a) shows a typical transport of sediment by advection with limited dampening, while figure $4(b-d)$ show the effect of the Péclet number and $R$ ratio, which cause the spreading of the initial pulse. In all of these three cases, aside from diffusion, advection and attenuation of the initial perturbation are also seen. This figure illustrates that the nature of bed load transport, whether it is dominated by advection or dispersion, is essentially dependent on the scale of observation (as is the case for other transport problems).

\subsection{Stochastic fluctuations}

We now revisit the sediment transport rate fluctuations. The major contrast to the method for an isolated control volume is that we now consider a current of particles 
across the array of cells. The displacement of a cloud of particles can be broken down into advection (at velocity $\bar{u}_{s}$ ) and diffusive spreading at a rate $\mathscr{D} \approx D_{u}$ (see the supplementary material for the proof). Here diffusion refers to the dispersal of particles arising from the difference between the actual particles' velocity and their mean velocity $\bar{u}_{s}$. Advection is a purely deterministic process and therefore does not cause any stochastic fluctuations and for this reason, we will discard it in the earlier developments below, but we will reintegrate it into the final governing equation. Stochastic fluctuations are thus created by particle diffusion as well as bed exchanges. In order to deal with particle diffusion with the same formalism as that used for tracking the evolution of the particle number in a control volume as a result of bed exchanges (see $\$ 3$ ), we consider particle diffusion as the limit of a local jump process (Gardiner 1983; Gillespie 1992), i.e. particles can be transferred from one cell $i$ to its neighbouring cells $i-1$ or $i+1$, centred at $x_{i-1}$ and $x_{i+1}$. This local jump process occurs at a rate $d_{i}=\mathscr{D}\left(x_{i}\right) / \Delta x^{2}$. The probability of one transfer per unit time is then $d_{i} N_{i}$ :

$$
N_{i} \stackrel{d_{i}}{\rightarrow} N_{i+1} \quad \text { and } \quad N_{i} \stackrel{d_{i}}{\rightarrow} N_{i-1},
$$

which is associated with the multivariate master equation

$$
\frac{\partial}{\partial t} P(\boldsymbol{n}, t)=\sum_{i} d_{i}\left\{\left(n_{i}+1\right)\left(P\left(\boldsymbol{n}+\boldsymbol{r}_{i}^{i+1}, t\right)+P\left(\boldsymbol{n}+\boldsymbol{r}_{i}^{i-1}, t\right)\right)-2 n_{i} P(\boldsymbol{n}, t)\right\},
$$

where $\boldsymbol{r}_{i}^{j}$ is a vector in which all but two entries are zero: $r_{i}=1, r_{j}=-1, r_{k}=$ 0 for $k \neq i, j$. Here $P\left(\boldsymbol{n}+\boldsymbol{r}_{i}^{i-1}, t\right)$ is thus the probability of observing the system jumping from a state $\boldsymbol{n}^{\prime}=\left(n_{1}, n_{2}, \ldots, n_{i-1}-1, n_{i}+1, n_{i+1}, \ldots\right)$ to a state $\boldsymbol{n}=$ $\left(n_{1}, n_{2}, \ldots, n_{i-1}, n_{i}, n_{i+1}, \ldots\right)$, i.e. there being a jump of one particle from cell $i$ to $i-1$ while the number of particles in other cells does not change. In this process, we do not consider the possibility that the particles' jump length exceeds $\Delta x$, which is a reasonable assumption given the fast decay in the probability distribution of the particle velocity (see the supplementary material). In the limit $\Delta x \rightarrow 0$ and making use of the second-order difference $f(n+1)+f(n-1)-2 f(n) \approx \Delta x^{2} f^{\prime \prime}(n)$ in (4.12), we find that (4.12) leads to a governing equation for $\gamma$ that is very close to (4.3)

$$
\frac{\partial}{\partial t} \gamma(x, t)=D_{u}(x, t) \frac{\partial^{2}}{\partial x^{2}} \gamma(x, t)
$$

as we have $D_{u}\left(x_{i}\right)=\mathscr{D}\left(x_{i}\right)$ (see the supplementary material). The important differences between (4.3) and (4.13) (once averaged) are that (i) advection is not considered (but it will be reintegrated below) and (ii) $D_{u}$ comes up as a weighting parameter of the diffusion term $\partial_{x x} \gamma$. In practice, we discard the contribution $\gamma \partial_{x x} D_{u}+2 \partial_{x} D_{u} \partial \gamma$ when using a Markov jump process instead of the Langevin model. This latter difference appears sufficiently minor for us to regard the two formulations as equivalent in the following.

System linearity makes it possible to combine the two master equations (4.12) and (3.1) into a single one that represents the $N$ variations due to particle diffusion and bed exchanges

$$
\frac{\partial}{\partial t} P=\sum_{i} d_{i}\left\{\left(n_{i}+1\right)\left(P\left(\boldsymbol{n}+\boldsymbol{r}_{i}^{i+1}, t\right)+P\left(\boldsymbol{n}+\boldsymbol{r}_{i}^{i-1}, t\right)\right)-2 n_{i} P(\boldsymbol{n}, t)\right\}
$$




$$
\begin{aligned}
& +\left(n_{i}+1\right) \sigma_{i} P\left(\boldsymbol{n}+\boldsymbol{r}_{i}^{+}, t\right)+\left(\lambda_{i}^{\prime}+\left(n_{i}-1\right) \mu_{i}\right) P\left(\boldsymbol{n}+\boldsymbol{r}_{i}^{-}, t\right) \\
& -\left(\lambda_{i}^{\prime}+n_{i}\left(\sigma_{i}+\mu_{i}\right)\right) P(\boldsymbol{n}, t),
\end{aligned}
$$

a where $\boldsymbol{r}_{i}^{ \pm}$is a vector in which all but one entries are zero: $r_{i}= \pm 1, r_{k}=0$ for $k \neq i$. The parameters $\lambda_{i}^{\prime}, \mu_{i}$ and $\sigma_{i}$ are the entrainment and deposition parameters for cell $i$.

As it stands, the set of equations (4.14) consists of strongly coupled partial differential equations as the arguments of $P$ involve not only $\boldsymbol{n}$ but also $\boldsymbol{n}+\boldsymbol{r}_{i}^{k}$ with $k= \pm 1$. It is too involved to be amenable to further analysis. As evoked in the introduction of this section, the trick is to use a Laplace-like transform, which makes it possible to pass from discrete to continuous probabilities. To that end, we use the Poisson representation introduced by Gardiner \& Chaturvedi (1977) in chemical physics, which expands any probability density function into a series of Poisson distributions with parameter $a_{i}$ (see appendix A and the supplementary material for further information):

$$
P(\boldsymbol{n}, t)=\prod_{i} \int_{\mathscr{C}} \frac{\mathrm{e}^{-a_{i}} a_{i}^{n}}{n !} f(\boldsymbol{a}, t) \mathrm{d} \boldsymbol{a},
$$

where $\boldsymbol{a}=\left(a_{i}\right)$ for $i=1,2, \ldots$ and $f(\boldsymbol{a}, t)$ is a multivariate pseudo-probability density function. This means that instead of working with the discrete random variable $\boldsymbol{n}$, we now work with the continuous random variable $\boldsymbol{a}=\left(a_{1}, a_{2}, \ldots\right)$. We end up with a governing equation for $f(\boldsymbol{a}, t)$

$$
\frac{\partial}{\partial t} f(\boldsymbol{a}, t)=\sum_{i} \mu_{i} \frac{\partial^{2} a_{i} f}{\partial a_{i}^{2}}+\frac{\partial}{\partial a_{i}}\left\{\left[d_{i}\left(a_{i+1}+a_{i-1}-2 a_{i}\right)+\lambda_{i}^{\prime}-a_{i}\left(\sigma_{i}-\mu_{i}\right)\right] f\right\} .
$$

The main advantages of this transformation appear here clearly: we have reduced the degree of coupling between the equations and the resulting set of equations is a multivariate advection diffusion equation that can be interpreted as a Fokker-Planck equation. Therefore, we can use the equivalence between Fokker-Planck equations and stochastic differential (Langevin) equations (Gardiner 1983). The Langevin representation equivalent to (4.16) is

$$
\mathrm{d} a_{i}(t)=\left(d_{i}\left(a_{i+1}+a_{i-1}-2 a_{i}\right)-\lambda_{i}^{\prime}-a_{i}\left(\sigma_{i}-\mu_{i}\right)\right) \mathrm{d} t+\sqrt{2 \mu_{i} a_{i}} \mathrm{~d} W_{i}(t),
$$

which holds for $i=2,3 \ldots$ and where $W_{i}$ is a Wiener process for cell $i$.

It is now very tempting to turn this set of coupled Langevin equations into a stochastic partial differential equation. As for stochastic integration, the procedure is not unique and admits variants with different physical meanings. We refer to other publications for alternative approaches in the context of reaction-diffusion processes (Dogan \& Allen 2011; Bulut \& Allen 2012); these approaches differ from each other in their expression of the noise term in the Langevin equation. Here, taking inspiration from Gardiner (1983), we define a Gaussian noise term $\xi(x, t)$ such that $\left\langle\xi_{b}(x, t) \xi_{b}\left(x^{\prime}, t^{\prime}\right)\right\rangle=\delta\left(x-x^{\prime}\right) \delta\left(t-t^{\prime}\right)$, i.e. a white noise term that is uncorrelated in time and space. To finalize our derivation, we take the continuum limit $\Delta x \rightarrow 0$ of (4.17) and introduce the continuous function $b(x, t)$ which takes on values on each cell as follows: $b\left(x_{i}, t\right)=a_{i}$. We end up with the following Langevin equation for $b(x, t)$ :

$$
\frac{\partial}{\partial t} b(x, t)=D_{u} \frac{\partial^{2} b}{\partial x^{2}}-\bar{u}_{s} \frac{\partial b}{\partial x}+\lambda^{\prime}(x, t)-\kappa(x, t) b+\sqrt{2 \mu b} \xi_{b},
$$


where $\kappa(x, t)=\sigma(x, t)-\mu(x, t)$. Note that we have reintegrated the advection contribution, which represents the transport of fluctuations, not their creation or destruction. Note also that equations with a multiplicative noise term such as the term on the right-hand side of (4.18) arise for autocatalytic chemical reactions (Schulz 2008).

The physical interpretation of (4.18) closely follows what is given for the one-cell configuration (see $\S$ A.5). We highlight the key results. In the absence of collective entrainment $(\mu=0)$, equation (4.18) is a classic partial differential (Liouville) equation in $b$, which implies that $b$ is no longer a random variable, but a deterministic process in the $b$-space. In the physical $\gamma$-space, this implies that all fluctuations are Poissonian. It comes as no surprise that for steady-state and perfectly homogeneous beds (no dependence on $x$ ), (4.18) shows that the steady solution is $\langle b\rangle_{s}=\lambda^{\prime} / \kappa$. For non-homogeneous cases, the Poisson rate $b(x, t)$ varies locally as a function of time depending on the various processes at play (exchanges with the bed, advection, diffusion). When collective entrainment occurs $(\mu>0)$, the dynamics of the fluctuations depart from the non-homogeneous Poisson case owing to the influence of the nonlinear square-root multiplicative noise $\sqrt{2 \mu b} \xi_{b}$ in (4.18).

If we take the average and use $\langle\gamma\rangle=\varpi_{p} \Delta x^{-1}\langle b\rangle$, we almost retrieve the non-homogeneous advection diffusion (4.5), with the main difference concerning the place of the diffusion coefficient $D_{u}$, a point that we discussed above for (4.13). Another important aspect related to the alternative approach involves starting from the mass and momentum conservation equations of continuum mechanics to derive a Langevin-like Exner equation, as for instance Jerolmack \& Mohrig (2005) did by adding a white noise term to their Exner equation. Here, our microstructural analysis shows that the structure of the noise term is more complicated as its strength is modulated by $\sqrt{2 \mu b}$, i.e. noise is amplified or self-sustaining for sufficiently high $b$ values, but may become very small when $b$ tends to 0 . This implies that the noise-induced pattern development may be more complicated than that shown by Jerolmack \& Mohrig (2005). Let us now take a closer look at pattern formation.

\subsection{Incipient development of bed patterns}

Pattern formation along the bed may result from the growth of spatial correlations. We assume that we start with a bed at equilibrium, which continues to evolve under steady conditions. To study the growth of the spatial correlations between two points $x$ and $x^{\prime}$, we introduce the spatial correlation function in the $b$-space

$$
g_{b}\left(x, x^{\prime}, t\right)=\left\langle b(x, t) b\left(x^{\prime}, t\right)\right\rangle-\langle b(x, t)\rangle\left\langle b\left(x^{\prime}, t\right)\right\rangle .
$$

To simplify the analysis, we assume that the system is in a homogeneous state and therefore, the correlation function depends solely on the distance $r=\left|x^{\prime}-x\right|$. By differentiating (4.19) with respect to time, and making use of (4.18), the change of variable $r=\left|x^{\prime}-x\right|,\left\langle\xi_{b}(x, t) \xi_{b}\left(x^{\prime}, t\right)\right\rangle=\delta\left(x-x^{\prime}\right)$, Itô's convention, after a few algebraic manipulations (see the supplementary material), we end up with the following governing equation for $g_{b}(r, t)$ :

$$
\frac{1}{2} \frac{\partial g_{b}}{\partial t}=D_{u} \frac{\partial^{2} g_{b}}{\partial r^{2}}-\kappa g_{b}+\mu \delta(r)\langle b\rangle,
$$

in which we note that the constant and convective terms have cancelled out. It is straightforward to deduce the steady-state solution using Fourier transforms (see the 
supplementary material). We find

$$
g_{b, s}(r)=\frac{1}{2} \frac{\mu\langle b\rangle_{s}}{\kappa \ell_{c}} \mathrm{e}^{-r / \ell_{c}},
$$

where $\langle b\rangle_{s}=\lambda^{\prime} / \kappa$ is the steady-state solution of (4.18) and $\ell_{c}=\sqrt{D_{u} / \kappa}=\sqrt{D_{u} t_{c}}$ is the correlation length. This latter parameter reflects the combined action of the water stream (through $D_{u}$ ) and sediment transport (through the deposition and entrainment rate difference $\kappa=\sigma-\mu$ ), a 'conspiration' from which emerges the correlation lengthscale $\ell_{c}$. Extending the relation (A 23) between the $p$-factorial moments of $N$ and their representation in the $a$-space, we may derive a continuum variant that links the moments of $\gamma$ and those of $b$ (see the supplementary material):

$$
\begin{aligned}
\langle\gamma(x, t)\rangle & =\varpi_{p} \Delta x^{-1}\langle b(x, t)\rangle, \\
\left\langle\gamma(x, t) \gamma\left(x^{\prime}, t\right)\right\rangle & =\varpi_{p}^{2} \Delta x^{-2}\left(\left\langle b(x, t) b\left(x^{\prime}, t\right)\right\rangle+\delta\left(x-x^{\prime}\right)\langle b(x, t)\rangle\right), \\
g_{\gamma}(r) & =\left\langle\gamma(x, t) \gamma\left(x^{\prime}, t\right)\right\rangle-\langle\gamma(x, t)\rangle\left\langle\gamma\left(x^{\prime}, t\right)\right\rangle .
\end{aligned}
$$

From these relations, we can infer the spatial correlation for $\gamma$

$$
\begin{aligned}
g_{\gamma, s}(r) & =\varpi_{p}^{2} \Delta x^{-1}\left(g_{b, s}(r)+\delta\left(x-x^{\prime}\right)\langle b\rangle_{s}\right), \\
& =\langle\gamma\rangle_{s} \frac{\varpi_{p}}{\Delta x}\left(\frac{1}{2} \frac{\mu}{\kappa \ell_{c}} \mathrm{e}^{-r / \ell_{c}}+\delta\left(x-x^{\prime}\right)\right),
\end{aligned}
$$

or, equivalently, its wavenumber spectrum

$$
S(k)=\langle\gamma\rangle_{s} \frac{\varpi_{p}}{\Delta x}\left(\frac{1}{\left(k \ell_{c}\right)^{2}+1} \frac{\mu}{\kappa}+1\right),
$$

where $k$ is the wavenumber. Figure 5 shows the wavenumber spectrum for various values of the ratio $\mu / \kappa$. In the absence of collective entrainment $(\mu=0)$, the spectrum is flat, meaning that there is no correlation in the particle activity in the homogeneous case (white noise). When collective entrainment occurs $(\mu>0)$, there are areas characterized by high correlations in the particle activity, whose strength is dictated by the ratio $\mu / \kappa$. This is likely to cause non-homogeneous sediment transport, which in turn promotes bed form development. From this perspective, the initiation of the bed form formation is the consequence of collective entrainment. The subsequent development of bed patterns is, however, beyond the scope of this analytical application as it requires calculating the coupling between the stream and topography, and more specifically the effects of turbulence on particle entrainment.

\section{Applications}

To provide proof of concept, we compare the main theoretical results with experimental data. These data come from earlier experimental campaigns conducted on steep slopes, thus in the supercritical regime (Böhm et al. 2004; Ancey et al. 2008; Heyman et al. 2013), but the theory applies to subcritical regimes as well. Only essential details of methodology, data processing and results are presented here. The supplementary material gives more information on these data and how theory performs when tested against the whole set of available data. In a companion paper, we will present a thorough comparison with experimental data in supercritical and subcritical flow conditions; emphasis is given to the spatiotemporal correlations of the particle flux, especially the role played by the correlation length $\ell_{c}$ (relative to the scale of observation) in the fluctuation characterization. 


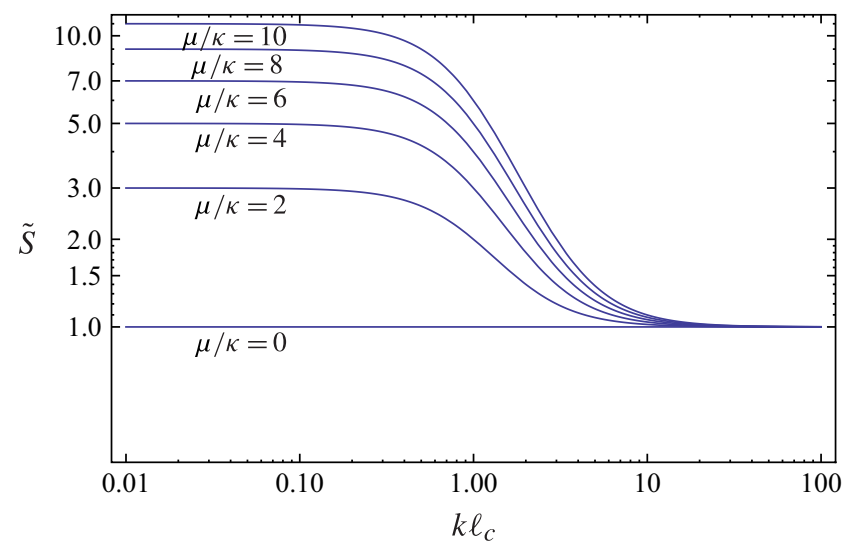

FIGURE 5. (Colour online) Variation of the scaled wavenumber spectrum $\tilde{S}(\tilde{k})=$ $S(k) /\left(\langle\gamma\rangle_{s} \varpi_{p}\right)$ where $\tilde{k}=k \ell_{c}$ for different values of the ratio $\mu / \kappa$.

\subsection{Probability density function of the particle velocity}

Here we take a look at the probability distribution of the streamwise component $u_{p}$ of the particle velocity. Experiments were conducted using a $8 \mathrm{~cm}$ wide flume, inclined at $\theta=1.6^{\circ}$ to the horizontal. The experimental set-up is presented in Heyman et al. (2013). The bed was composed of gravel characterized by a narrow size distribution around a mean diameter $8 \mathrm{~mm}$. The particle density was $2650 \mathrm{~kg} \mathrm{~m}^{-3}$. Particle motion was tracked using a high-speed camera over a $40 \mathrm{~cm}$ length (Phantom v640 operated at $150 \mathrm{~Hz}$ with a field of $800 \times 104$ pixels). Trajectories of individual particles were captured using a particle tracking method based on the Matlab 'polyparticletracker' routines (Rogers, Waigh, Zhao \& Lu 2007). Figure 6 shows the probability density function of the particle velocity $u_{p}$ computed from 755 trajectories. The flow conditions were the following: depth-averaged velocity of water $\bar{v}=92.5 \mathrm{~cm} \mathrm{~s}^{-1}$, mean flow depth $h=2 \mathrm{~cm}$, Froude number $F r=\bar{v} / \sqrt{g h}=2.1$, flow Reynolds number $R e=\bar{v} h / v_{w}=18 \times 10^{3}\left(v_{w}\right.$ kinematic viscosity), Shields number $S h=\varrho h \sin \theta /\left[\left(\varrho_{p}-\varrho\right) d\right]=0.042$. For these flow conditions, long-wavelength bed forms developed. Figure 6 also shows the theoretical probability distribution (3.10), whose parameters were fitted using the method of moments: $\bar{u}_{s}=29.9 \mathrm{~cm} \mathrm{~s}^{-1}$ and $\zeta=\bar{u}_{s} \sqrt{t_{r} / D_{u}}=5.7$. Additional tests (PP and QQ plots) are presented in the supplementary material.

There is a fairly good agreement between these data and the truncated Gaussian distribution (3.10). Taking a closer look at the tail of the distribution reveals, however, that the theoretical distribution overestimates the velocity quantiles for $u_{p}>1 \mathrm{~m} \mathrm{~s}^{-1}$ (i.e. when the particles moved faster than the mean water flow). This result compares well with the observations made by Martin, Jerolmack \& Schumer (2012) with similar flow conditions $\left(7 \mathrm{~mm}\right.$ particles, mean particle velocity ranging from 0.3 to $0.8 \mathrm{~m} \mathrm{~s}^{-1}$, water velocity in the $0.6-0.8 \mathrm{~m} \mathrm{~s}^{-1}$ range, bed inclination of $5^{\circ}$ ). They also found that the Gaussian distribution closely matched the empirical probability distribution of the velocity components.

Our results contrast with those obtained by Roseberry et al. (2012), Furbish et al. (2012b) and Lajeunesse et al. (2010), who found that the empirical probability distribution of particle velocity $u_{p}$ was well captured by an exponential distribution. This discrepancy may originate from the differences in the experimental set-up. Indeed, 


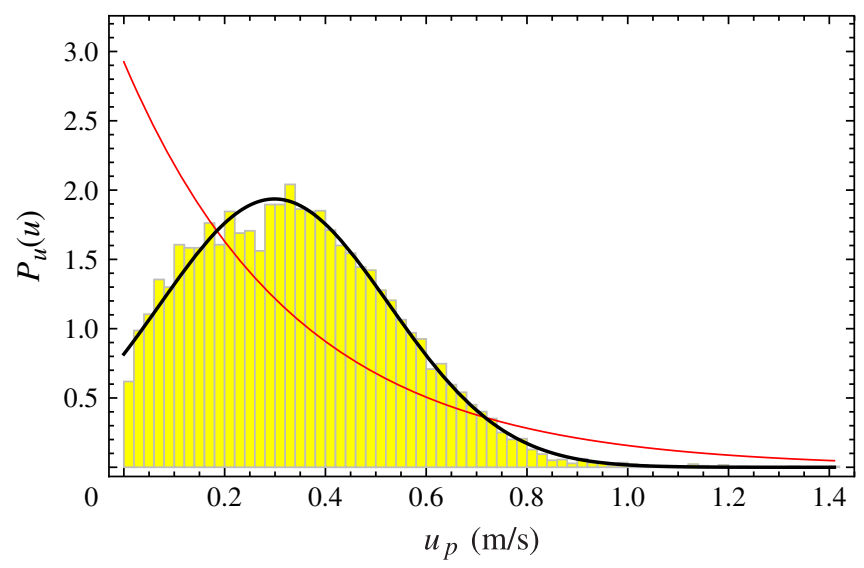

FIgURE 6. (Colour online) Probability distribution for the particle velocity. The histogram represents the empirical probability density function of $u_{p}$. The thick (black) solid line is the theoretical distribution (3.10) with $\bar{u}_{s}=29.9 \mathrm{~cm} \mathrm{~s}^{-1}$ and $\zeta=5.7$. The thin (shown in red online) line shows the exponential probability distribution $P_{u}(u)=\mathrm{e}^{-u / \bar{u}_{s}} / \bar{u}_{s}$, still with $\bar{u}_{s}=29.9 \mathrm{~cm} \mathrm{~s}^{-1}$.

these authors used fine sand: $0.5 \mathrm{~mm}$ for Roseberry et al. (2012) and 1-2 mm for Lajeunesse et al. (2010). Moreover, the flows were subcritical: $F r \sim 0.3$ for Roseberry et al. (2012) and $F r<0.95$ for Lajeunesse et al. (2010). For Roseberry et al. (2012), the mean particle velocity was in the $3-5 \mathrm{~cm} \mathrm{~s}^{-1}$ range, yielding an estimated ratio $\bar{u}_{s} / \bar{v}$ lower than 0.15 , i.e. at relatively low sediment transport rates (whereas we had $\bar{u}_{s} / \bar{v} \sim 0.3$ in our experiments).

Although there is experimental evidence for the theoretical velocity distribution (3.10), the diversity of experimental data shows that its range of application is unlikely to cover all sediment sizes. We note, however, that in either case, the truncated Gaussian and exponential laws are thin-tailed, a result that can be anticipated as it is uncommon for the highest particle velocities to exceed fluid velocities. Therefore, the high fluctuations of sediment transport rates are unlikely to stem from a thick tail of the velocity distribution.

\subsection{Fluctuations of the particle flux in the absence of bed exchanges}

Here we address the case of sediment transport over a fixed corrugated bed. There is thus neither entrainment nor deposition $\left(\lambda^{\prime}=\mu=\sigma=0\right)$. The experimental set-up is presented in Böhm et al. (2004).

Experiments were conducted using a $6.5 \mathrm{~mm}$ wide flume, inclined at $\theta=5^{\circ}$. The bed was composed of an array of half-cylinder of equal size $(3 \mathrm{~mm})$. The moving particles were glass beads with diameter $6 \mathrm{~mm}$. Their density was $2500 \mathrm{~kg} \mathrm{~m}^{3}$. Particles were fed into the flume at an inflow rate $\dot{n}_{0}=8$ beads $\mathrm{s}^{-1}$. Particle motion was tracked over a $22.5 \mathrm{~cm}$ length using a high-speed camera (Pulnix TM-6505 AN operated at $130 \mathrm{~Hz}$ with a field of $640 \times 192$ pixels). Figure 7 shows the probability density function of the particle flux $\dot{n}$ obtained from a sequence of 8000 frames (i.e. over about $1 \mathrm{~min}$ ). The flow conditions were the following: depth-averaged velocity of water $\bar{v}=53 \mathrm{~cm} \mathrm{~s}^{-1}$, mean flow depth $h=1 \mathrm{~cm}$, Froude number $F r=1.7$, flow Reynolds number $R e=5300$ and Shields number $S h=0.11$. 


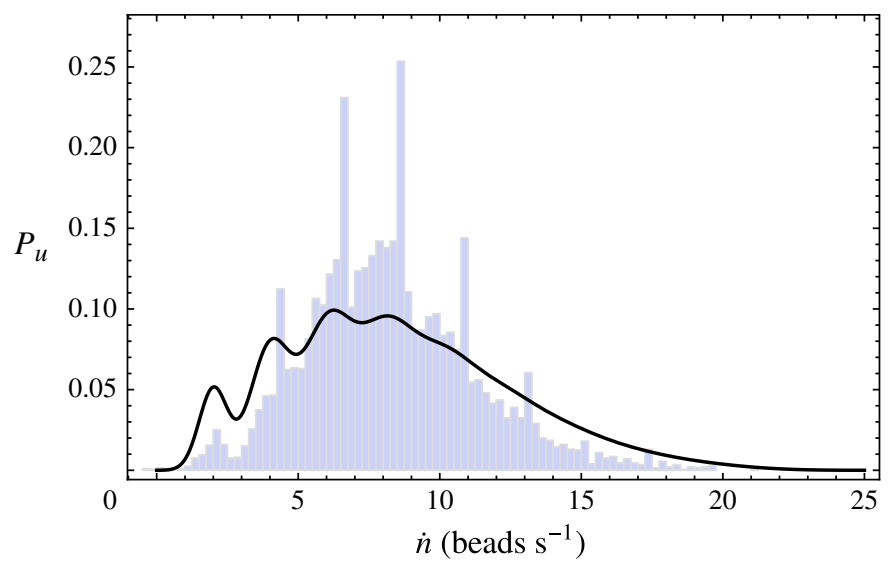

FIgURE 7. (Colour online) Probability density function of the particle flux. The histogram represents the empirical probability density while the solid line is the theoretical distribution (B 2) with $\bar{u}_{s}=44 \mathrm{~cm} \mathrm{~s}^{-1}$ and $\zeta=2.71$.

Figure 7 also shows the theoretical probability distribution $P_{\dot{n}}$ (B 2), whose parameters were obtained by averaging the trajectory features: $\bar{u}_{s}=44 \mathrm{~cm} \mathrm{~s}^{-1}$ and var $u=10^{-2} \mathrm{~cm}^{2} \mathrm{~s}^{-2}$, yielding $\zeta=2.71$ (Böhm et al. 2004). Additional tests (PP and QQ plots) are presented in the supplementary material. The theoretical distribution (B 2) captures the main trends, but taking a closer look at the data (the cumulative distribution function and QQ plot, shown in the supplementary material) reveals that there is a systematic deviation between the empirical and theoretical distributions. The main reason for this discrepancy is the slight deviation from the expected Poissonian distribution for the number of moving particles (see the supplementary material). Theory requires that the variance $\operatorname{var} N$ be equal to the mean value $\langle N\rangle$, but experimentally we found that the variance of $N$ was twice as small as the mean value: $\operatorname{var} N=2.1$ but $\langle N\rangle=4.2$. This was probably the consequence of the supply system (consisting of a star wheel injecting particles at constant rate) and the short length of the flume $(2 \mathrm{~m})$, leading to overly steady particle arrivals. Spikes are conspicuous in both the empirical and theoretical probability distributions. They result from the finite-size effects of the observation window: each spike corresponds to a finite number of particles within the observation window. Theory tends to smear out the spike amplitude for $\dot{n} \geqslant 5$ beads $\mathrm{s}^{-1}$ whereas experiments exhibited marked peaks for particle fluxes as large as 13 beads $\mathrm{s}^{-1}$.

\subsection{Fluctuations of the particle flux as a result of bed exchanges}

The last application concerns the transport of particles with exchanges with the bed. The experimental set-up and data are presented by Ancey et al. (2006) and Ancey et al. (2008). The flume configuration is identical to that presented in $\$ 5.2$ except that now there is a bed composed of the same beads as those injected at the flume inlet. Here we present some data obtained with a flume inclination of $\theta=5^{\circ}$ and varying inflow rate $\dot{n}_{0}$ (from 5 to 20 beads s$^{-1}$ ). All of the data drawn from Ancey et al. (2008) are shown in the supplementary material. Table 1 summarizes the features of the four experiments presented here.

Figure 8 shows the empirical probability density function $P_{n}(n)$ and the theoretical distribution, the negative binomial law (3.2) with parameters given by (3.3) and (3.4). 


\begin{tabular}{|c|c|c|c|c|}
\hline Run name & (e) & (g) & (i) & (j) \\
\hline$\dot{n}_{0}\left(\right.$ beads $\left.\mathrm{s}^{-1}\right)$ & 5.3 & 8.0 & 15.4 & 20.0 \\
\hline$h(\mathrm{~mm})$ & 10.2 & 12.2 & & 19.4 \\
\hline $\bar{v}\left(\mathrm{~m} \mathrm{~s}^{-1}\right)$ & .41 & 0.44 & .48 & 0.53 \\
\hline $\operatorname{Re}$ & 20 & 4550 & 280 & 910 \\
\hline$F r$ & & 1.38 & & .26 \\
\hline$S h$ & 11 & 0.13 & .19 & 0.21 \\
\hline $\bar{u}_{r o l}\left(\mathrm{~m} \mathrm{~s}^{-1}\right)$ & .06 & .06 & .08 & 0.07 \\
\hline $\bar{u}_{s a l}\left(\mathrm{~m} \mathrm{~s}^{-1}\right)$ & .28 & 0.29 & .32 & 0.32 \\
\hline $\left.\mathrm{s}^{-1}\right)$ & 0.14 & 0.13 & 0.15 & 0.14 \\
\hline$E$ (beads $\mathrm{s}^{-1}$ ) & 48.5 & 681 & 103 & 150 \\
\hline$D$ (beads $\left.\mathrm{s}^{-1}\right)$ & & & & 151 \\
\hline$\langle N\rangle$ & 9.46 & 13.8 & 22.8 & 34.2 \\
\hline $\operatorname{var} N$ & 70.7 & 64.5 & 72.1 & 128 \\
\hline$\langle\dot{n}\rangle$ (beads $\mathrm{s}^{-1}$ ) & 5.72 & 7.74 & 15.6 & 20.6 \\
\hline$\sigma\left(\mathrm{s}^{-1}\right)$ & 5.12 & 4.95 & 4.52 & 4.39 \\
\hline & 4.42 & 3.89 & 3.09 & 3.22 \\
\hline$x^{\prime}\left(\mathrm{s}^{-1}\right)$ & 6.48 & 14.5 & 32.7 & 39.9 \\
\hline
\end{tabular}

TABLE 1. Flow characteristics for experiments (e), (g), (i), and (j) carried out at $\theta=5^{\circ}$. Varying parameter: inflow rate $\dot{n}_{0}$ (imposed at the flume inlet). Water flow characteristics $\bar{v}$ and $h$ and time-averaged values of the Reynolds, Froude and Shields numbers characterizing bed load and water flow. For each experiment, we measured the particle velocity $\bar{u}_{r o l}$ in the rolling regime, their velocity $\bar{u}_{s a l}$ in the saltating regime, and the mean particle velocity $\bar{u}_{s}$. We also measured the mean number of deposited/entrained particles and then deduced the entrainment and deposition rates $E$ and $D$. We also counted the mean number of moving particles $\langle N\rangle$ as well as its variance var $N$. Using (2.2) with $\Delta x=22.5 \mathrm{~cm}$, we deduced the mean particle flux $\langle\dot{n}\rangle$ in the control volume. From the deposition and entrainment rates together with the number of moving particles, we inferred estimates of the entrainment and deposition parameters $\mu, \lambda^{\prime}$ and $\sigma$. See the supplementary material for the full set of experiments.

There is remarkably good agreement between data and theory for all experiments (see also the supplementary material). Compared with our earlier findings (Ancey et al. 2008), this result shows that we can remove the emigration/immigration process (which was our first attempt to model the advection and dispersal of a particle current) without altering the performance of the model. As we have investigated steady uniform flow conditions, this behavioural similarity between the two formulations is not really a surprise.

Figure 9 shows the empirical and theoretical probability density functions $P_{\dot{n}}(\dot{n})$. In that case, concordance between theory and experiments is less marked for the experiments presented here; in the supplementary material, we show that much better agreement is obtained at steeper slopes. One possible reason for this discrepancy is the existence of two populations of moving particles with two distinctive mean velocities. As shown by table 1 , there is a size factor of about five between the velocities in the rolling and saltating regimes. Comparing the different runs also shows that the larger the number of moving particles, the better the agreement. This may be an indication either that theory performs less well in the limit $N \rightarrow 0$ or the computation of the sediment transport rate developed in the supplementary material is biased as we assumed that the probabilities $P_{n}(n)$ and $P_{u}\left(u_{p}\right)$ were independent 


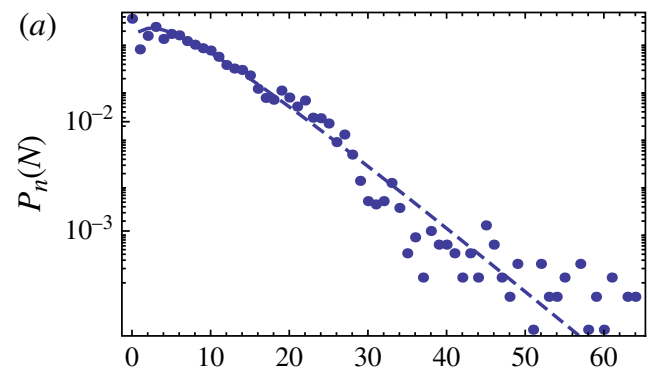

(b)
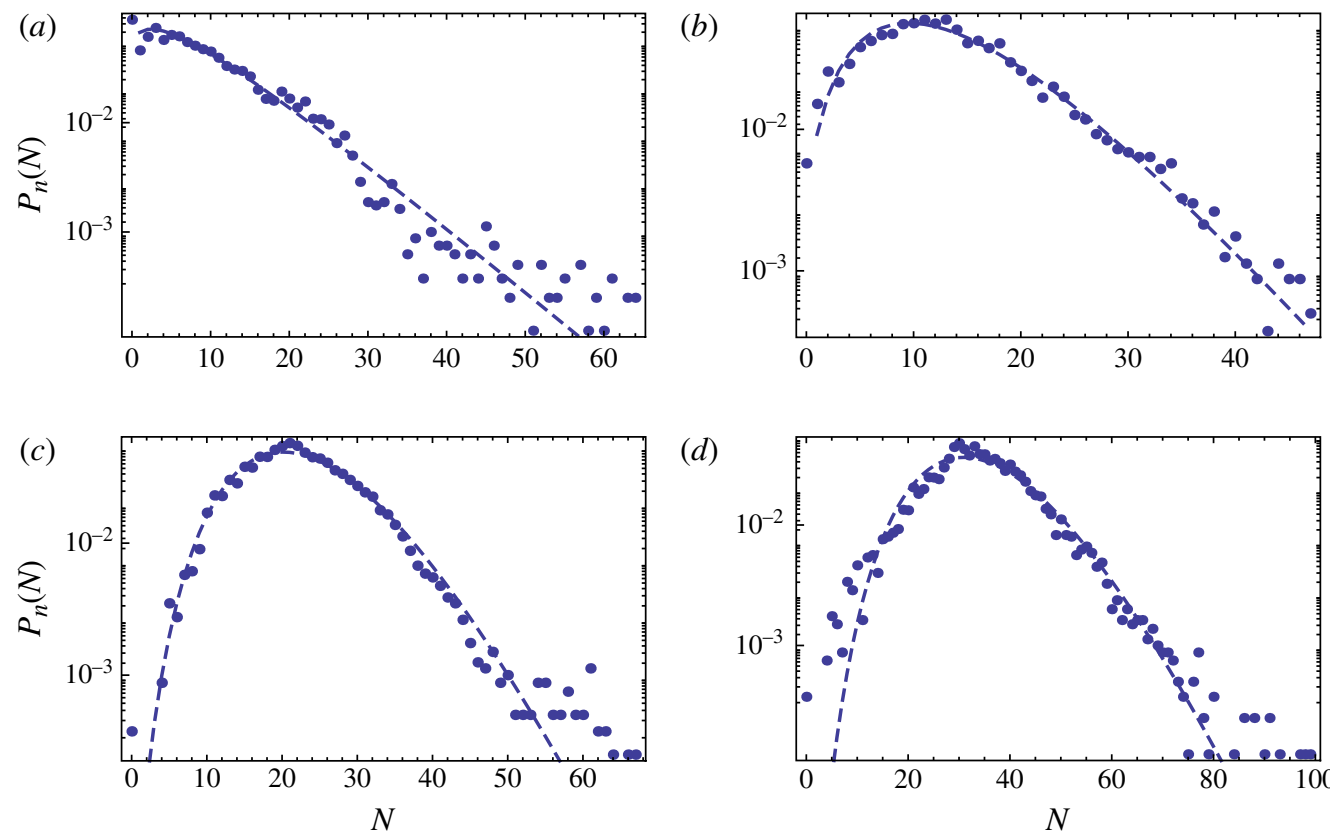

$(d)$

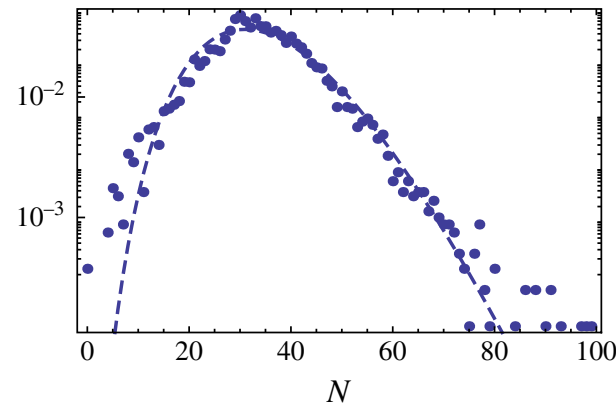

FIGURE 8. (Colour online) Probability density functions for the number of moving particles in experiments (e), (g), (i) and (j). The dots represent the empirical probability density while the dashed curves are the theoretical distributions (3.2).

in order to obtain $P_{\dot{n}}(\dot{n})$ by taking the Fourier transform of the convolution product. This requires further work.

\section{Concluding remarks}

This paper is a first stab at modelling the transport of coarse particles by a turbulent water stream, considering four processes in the particle motion: advection, dispersion, entrainment and deposition. Although in reality, water flow and sediment transport are interrelated in a complex nonlinear way, we have assumed in a first step that water is an external control parameter that drives sediment transport, but is not significantly affected in turn by the variations in the particle flux or bed elevation (see the discussion on the Saint-Venant-Exner equations in $\S 1$ ). As a consequence, all background information on turbulence structure and effects on particle entrainment has been implicitly summarized in the flow resistance and entrainment/deposition parameters. This assumption is more or less realistic in the absence of bed forms or intense sediment transport, two mechanisms that produce a strong interplay between the water and sediment phases (Powell 1998). This working assumption enables us to derive analytical results. Including phase interdependency would require numerical simulations (see the last paragraph of this section). Another simplification is the assumption of unidirectional flow. Formally, extension of the model to two-dimensional configurations is possible, but again requires numerical simulations. Finally, we have ignored variations in particle size, therefore leaving aside all of the problems related to grain sorting, hiding effects and bed armouring (Parker 2008; Wilcock, Pitlick \& Cui 2009). 

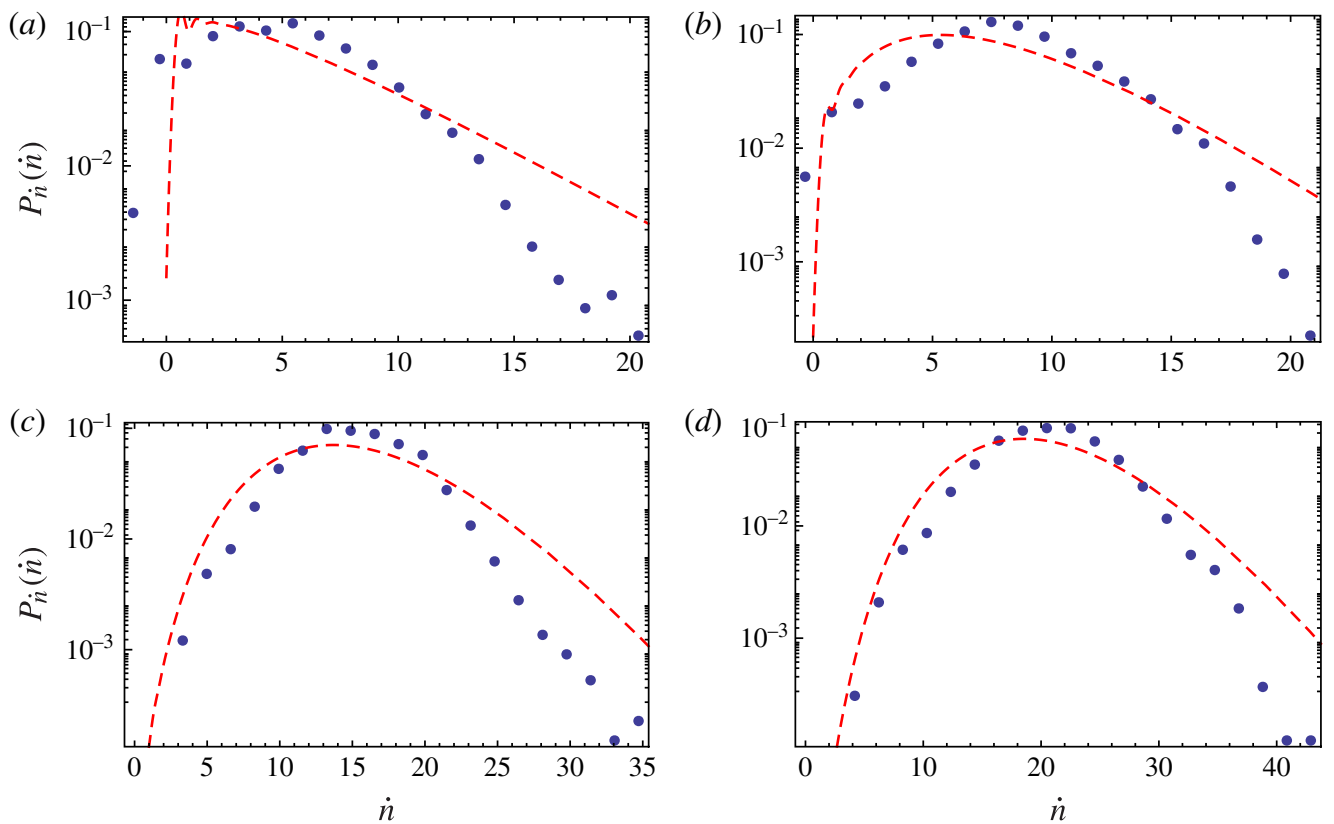

FIGURE 9. (Colour online) Probability density functions for the particle flux in experiments (e), (g), (i) and (j). The dots represent the empirical probability density function while the dashed curves are the theoretical distributions (B2) with $\zeta=4$. This parameter was fixed arbitrarily, but provided that $\zeta>3$, we found that altering $\zeta$ did not change the shape of $P_{\dot{n}}(\dot{n})$ significantly for these runs.

The four processes involved in the particle transport are associated with four characteristic times: $t_{c}=1 /(\sigma-\mu)$ is the time pertaining to the exchanges between bed and stream. Notably, it is also the autocorrelation time for the evolution of the number of moving particles for steady-state flow conditions. Particle dispersion is characterized by two times: $t_{d}=\Delta x^{2} / D_{u}$ (diffusion time) and $t_{r}$ (relaxation time). As usual, advection is best described by the time necessary for a particle to cross the control volume of length $\Delta x: t_{a}=\Delta x / \bar{u}_{s}$. Two dimensionless numbers have emerged from our analysis: the classic Péclet number $P e=t_{a} / t_{d}=\bar{u}_{s} \Delta x / D_{u}$ and the dimensionless group $\zeta=\bar{u}_{s} \sqrt{t_{r} / D_{u}}$. Our model breaks down in the limit $\zeta \rightarrow 0$. Indeed, in this limit, the forcing exerted by the water stream is not sufficient to impose the mean advection velocity $\bar{u}_{s}(\bar{v}, h)$ on the particles; as shown by figure 3 , the mean particle velocity at equilibrium differs significantly from $\bar{u}_{s}$ when $\zeta<1$. Another critical point is the asymmetric distribution of particle displacements when $\zeta<2$, leading to anisotropic particle diffusion (see the supplementary material). The domain $\zeta<2$ may correspond to flow conditions associated with incipient particle motion, but even for these conditions, it is unclear whether the mean particle velocity can be arbitrarily small or whether instead, particles set in motion reach a velocity that systematically exceeds a certain threshold. Available experimental data do not provide any evidence for the former possibility and if this holds true more generally, the $\zeta<2$ domain may be unattainable in practice. As illustrated by figure $10, \zeta$ influences the shape of the probability distribution of the particle flux $P_{\dot{n}}$. Smaller $\zeta$ values lead to smoother probability distributions. This parameter has, however, little influence on the mean and variance of the distribution $P_{\dot{n}}$. Although rarely used in 


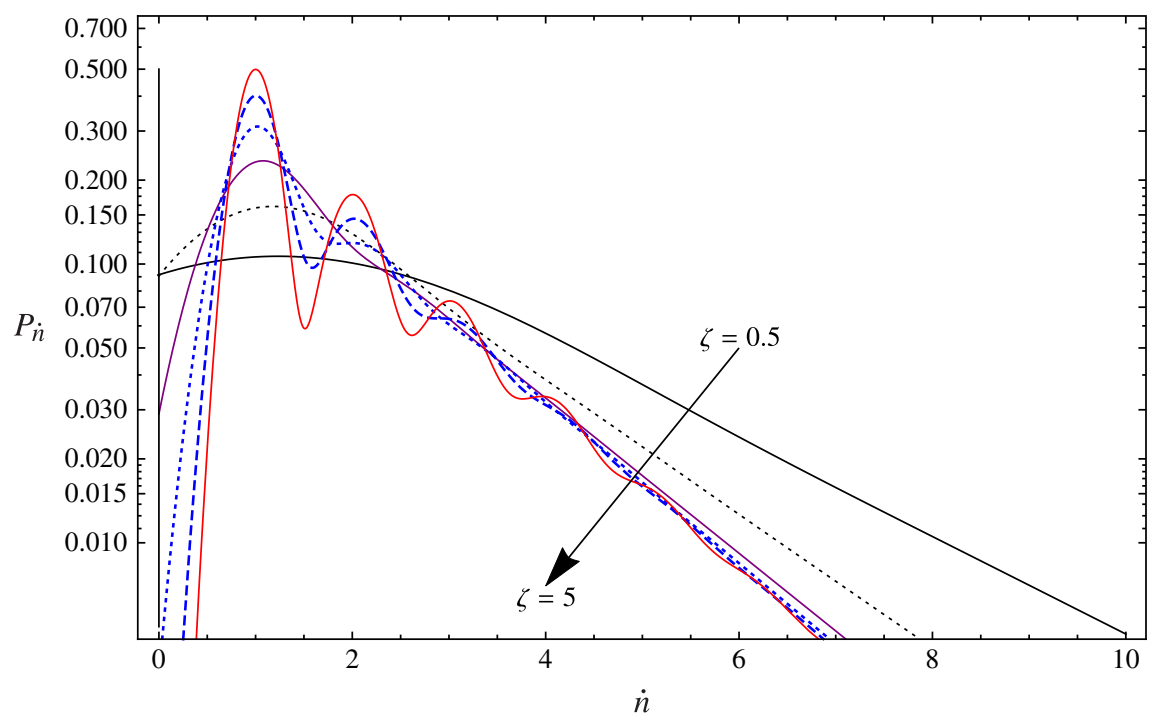

FIGURE 10. (Colour online) Shape of the probability density function $P_{\dot{n}}(\dot{n})$ given by (B 2) in a log-linear plot for different values of $\zeta$ (the arrow shows increasing $\zeta$ values): $\zeta=0.5$ (black solid line), $\zeta=1$ (black dotted line), $\zeta=2$ (purple solid line), $\zeta=3$ (blue dotted line), $\zeta=4$ (blue dashed line) and $\zeta=5$ (red solid line). All of the other parameters are kept constant: $\Delta x=1 \mathrm{~m}, \bar{u}_{s}=1 \mathrm{~m} \mathrm{~s}^{-1}, r=1\left(\lambda^{\prime}=\mu\right)$ and $p=0.5\left(\sigma=2 \lambda^{\prime}\right)$. The mean particle flux $\langle\dot{n}\rangle$ is $1.541,1.115,1.007,1.000,1.000$ and 1.000 beads $\mathrm{s}^{-1}$ when $\zeta$ is increased from 0.5 to 5 . The square coefficient of variation $\operatorname{var} \dot{n} /\langle\dot{n}\rangle^{2}$ is $2.410,2.362$, $2.209,2.109,2.062$ and 2.040 beads $^{2} \mathrm{~s}^{-2}$ when $\zeta$ is increased from 0.5 to 5 .

the context of sediment transport, the Péclet number is instrumental in the assessment of diffusion-induced effects on the particle flux. This effect has been documented through the typical problem of advection and scattering from an instantaneous source of sediment (see figure 4).

One particularly interesting result is related to the advection diffusion equation (4.3) that governs the variations in the mean particle activity $\langle\gamma\rangle$. We come to conclusions similar to those drawn by Furbish et al. (2012a) about the form that the particle flux should take. The governing equation (4.3), derived from the microscopic description of particle transport, matches the Exner equation (1.3) provided that (i) the particle transport rate $\bar{q}_{s}$ is defined at the macroscopic scale as

$$
\mathscr{Q}(x, t)=\langle\gamma\rangle \bar{u}_{s}-\frac{\partial}{\partial x}\left(D_{u}\langle\gamma\rangle\right)
$$

and (ii) the term $\partial_{t}\langle\gamma\rangle$ is negligible relative to entrainment and deposition rates. The latter assumption is well-established (Cui et al. 2005). Using a Lagrangian approach, Furbish et al. (2012a) obtained a slightly different equation: $\mathscr{Q}(x, t)=$ $\left\langle\gamma u_{p}\right\rangle-\frac{1}{2} \partial_{x}\left(D_{p}\langle\gamma\rangle\right)$, where $D_{p}$ denotes the particle diffusivity and is related to the second moment of particle displacements instead of the velocity fluctuations like in our approach. The difference, which lies in the factor $\frac{1}{2}$ weighting the spatial gradient, results essentially from a different notation convention, see (3.8) where the velocity diffusivity was (arbitrarily) weighted by a factor of two. As a consequence, the results of the present stochastic analysis closely follow those obtained by Furbish 
et al. (2012a): even at the macroscopic scale, diffusive effects are present in the Exner equation, which modulate, to a varying degree, the advection term.

The existence of diffusive effects in the Exner equation may lead to the conclusion that by smoothing out particle activity variations $\langle\gamma(x, t)\rangle$ along the bed, particle fluctuations dissipate short-wavelength perturbations and so make the bed more stable. Yet, as exemplified by Turing patterns in certain reaction-diffusion systems such as the Belousov-Zhabotinsky reaction in chemistry (Hoyle 2006), diffusion may amplify instabilities instead of dampening them under a slight perturbation by noise. Stability analysis goes far beyond the scope of this paper, but we keep in mind that upon studying the fluctuations of the number of moving particles $N$ in $\S 3$, we interpreted the steady-state probability distribution of $N$ as a Poisson distribution, whose parameter $r_{p}$ evolved randomly. When working in the probability $a$-space for an isolated control volume (see $\S$ A.4), we have found that the Poisson parameter $a$ fluctuates randomly around an asymptotic position $a_{\infty}$, with a finite variance given by (A 32) in the limit of $t \rightarrow \infty$ : var $a=\mu a_{\infty} / \kappa$. This variance is controlled by the collective entrainment parameter $\mu$. In particular, var $a$ may become arbitrarily high when $\kappa \rightarrow 0$, i.e. when $\mu \rightarrow \sigma$. The present analysis substantiates our previous findings: in the absence of collective entrainment $(\mu=0)$, the fluctuations are Poissonian, which leads to a rather simple macroscopic behaviour (Ancey et al. 2008; Heyman et al. 2013). In contrast, for $\mu>0$, fluctuations are non-Poissonian and may vary significantly over time, affecting the macroscopic behaviour by the growth of spatial correlations, which reflects local increases in the particle activity. Even for steady uniform flow conditions (with no bed forms), the variance and the coefficient of variation (B 5) of the particle flux may become very large. For time-dependent flow conditions and especially when bed forms migrate, the expected behaviour of fluctuations is quite complicated. The generalized Langevin equation (4.18) reveals that the noise structure, characterized by a square multiplicative noise term, differs significantly from the white noise term used by Jerolmack \& Mohrig (2005) to model the stochastic development of bed forms. Altogether, the Langevin equation (4.18) provides us with little reason to believe that in real flow conditions, marked by time dependence and bed form migration, one can obtain consistent time averages of the particle activity and sediment transport rates. In our opinion, this explains the failure in both the laboratory (Singh et al. 2009) and the field (Bunte \& Abt 2005; Recking et al. 2012) to arrive at robust estimates of transport rates when the sampling rate is changed.

Lastly, it is worth noting that the theoretical framework presented here is fairly easy to implement numerically compared with other stochastic methods for reaction-diffusion problems. Numerical simulations of multivariate master equations are fraught with difficulty and are relatively slow (Allen 2007; Gillespie 2007; Hita \& Ortiz de Zárate 2013). The strength of the Poisson representation used here is that by working in a continuous probability distribution space, we handle Langevin stochastic differential equations instead of multivariate master equations. There are a number of numerical techniques that perform well for Langevin equations with multiplicative noise (Dornic, Chate \& Munoz 2005; Iacus 2008). Here, the governing equations of the Poisson rates $a$ in (A 29) or $b$ or (4.18) belong to the class of Feller or Cox-Ingersoll-Ross processes, which have attracted considerable attention (Iacus 2008). The availability of standard numerical methods opens up considerable possibilities for the numerical solution of the Saint-Venant equations (1.1)-(1.2) coupled to a stochastic Exner equation. 


\section{Acknowledgements}

The work presented here was supported by the Swiss National Science Foundation under grants No. 200021-129538 (a project called 'The Stochastic Torrent: stochastic model for bed load transport on steep slope') and No. 200021-105193/1 (a project called 'Transient free-surface flows of concentrated suspensions - Application to geophysical flows', funded by an R'Equip grant). The project is part of a longer programme spanning several years and which has benefited from feedback and many discussions. We are indebted to our colleagues Dr Eric Bardou and Eric Travaglini from CREALP (Sion, Switzerland), Professor Anthony Davison (EPFL), Professor Patricio Bohorquez (University of Jaén, Spain), and Dr Philippe Frey (IRSTEA, France). Most of the data presented here was obtained by my former $\mathrm{PhD}$ student Tobias Böhm during his thesis. We are grateful to Belinda Bates for copy-editing the paper and Professor Anthony Davison for his remarks. We thank Professor Chris Paola for his steady support and constructive remarks, which helped us to improve the initial manuscript. Two anonymous reviewers provided thorough reviews and useful comments.

\section{Supplementary data}

Supplementary data is available at http://dx.doi.org/10.1017/jfm.2014.074.

\section{Appendix A. Poisson representation in the one-cell configuration}

\section{A.1. Erosion and sedimentation}

Because we will use the same mathematical tools as those used in the stochastic formulation of chemical kinetics, we draw an analogy between chemical equations and the exchanges between the bed and the stream. Our problem is tantamount to studying two chemical species: the moving particles $\mathrm{M}$ and the bed particles B. For deposition, we envision two mechanisms: (i) a moving particle $\mathrm{M}$ can come to rest at rate $\sigma_{0}$ and thus be transformed into a bed particle $\mathrm{B}$, (ii) a trajectory of a moving particle $\mathrm{M}$ can be disturbed by a bed particle, transforming $\mathrm{M}$ into a bed particle $\mathrm{B}$ at rate $\sigma_{1}$. We can summarize these transformations as

$$
\mathrm{M} \stackrel{\sigma_{0}}{\rightarrow} \mathrm{B} \quad \text { or } \quad \mathrm{M}+\mathrm{B} \stackrel{\sigma_{1}}{\rightarrow} \mathrm{B}+\mathrm{B}
$$

Note that we have no means of differentiating the different deposition processes occurring at rates $\sigma_{1}$ and $\sigma_{0}$. We then pose $\sigma=\sigma_{0}+\sigma_{1}$ and treat sedimentation as a single process. Similarly, we consider two mechanisms for entrainment. The first mechanism reflects the usual scenario of particle entrainment as a result of the fluid action. A bed particle can be entrained by the fluid and set in motion at rate $\lambda^{\prime}$

$$
\mathrm{B} \stackrel{\lambda^{\prime}}{\rightarrow} \mathrm{M}
$$

The second mechanism considers that a moving particle can interfere at rate $\mu$ with a bed particle and entrain it

$$
\mathrm{M}+\mathrm{B} \stackrel{\mu}{\rightarrow} \mathrm{M}+\mathrm{M}
$$

We refer to the former mechanism as individual entrainment and to the latter as collective entrainment. Some words of explanation are needed to justify the use of 
'collective.' The probability that a single moving particle dislodges a bed particle when impacting the bed (or by modifying the fluid stress field, which in turn destabilizes a bed particle) during an increment of time $\Delta t$ is $\mu \Delta t$. Since there are $N=n$ independent particles at time $t$, then using the addition law of probability, we deduce that the resulting probability is $\mu n \Delta t$. For individual entrainment, the probability is $\lambda^{\prime} \Delta t$ regardless of the number of particles in the cell.

Let us now translate these exchange rules into statistical terms. We consider the following exchanges over the time increment $\Delta t$, which is assumed to be sufficiently small that two events cannot occur in $(t, t+\Delta t)$. The corresponding transition probabilities of entrainment (birth) are respectively

$$
P(n \rightarrow n+1, \Delta t)=\lambda^{\prime} \Delta t+o(\Delta t), \quad P(n \rightarrow n+1, \Delta t)=\mu n \Delta t+o(\Delta t) .
$$

A moving particle can come to rest within the window, at rate $\sigma$ for each moving particle (death). The transition probability is thus

$$
P(n \rightarrow n-1, \Delta t)=n \sigma \Delta t+o(\Delta t) .
$$

With these assumptions and the discrete Chapman-Kolmogorov equation

$$
P(n, t+\Delta t)=\sum_{-\infty}^{+\infty} P(n+i, t) P(n+i \rightarrow n, \Delta t),
$$

we obtain a set of equations

$$
\begin{aligned}
P(n, t+\Delta t)= & \sigma(n+1) \Delta t P(n+1, t)+P(n-1, t)\left\{\lambda^{\prime}+(n-1) \mu\right\} \Delta t \\
& +P(n, t)\left\{1-\Delta t\left(\lambda^{\prime}+n \sigma+n \mu\right)\right\}+o(\Delta t),
\end{aligned}
$$

for $n=1,2 \ldots$, and

$$
P(0, t+\Delta t)=\sigma P(1, t) \Delta t+P(0, t)\left(1-\lambda^{\prime} \Delta t\right)+o(\Delta t),
$$

for $n=0$. Upon rearranging the terms and letting $\Delta t \rightarrow 0$, we obtain the forward master equation

$$
\begin{aligned}
\frac{\partial}{\partial t} P(n, t)= & (n+1) \sigma P(n+1, t)+\left(\lambda^{\prime}+(n-1) \mu\right) P(n-1, t) \\
& -\left(\lambda^{\prime}+n(\sigma+\mu)\right) P(n, t), \\
\frac{\partial P(0, t)}{\partial t}= & \sigma P(1, t)-\lambda^{\prime} P(0, t) .
\end{aligned}
$$

At time $t=0$, there are $N=N_{0}$ particles within the control volume, so we set

$$
P(n, 0)=\delta\left(n-N_{0}\right),
$$

where $\delta$ denotes the Kronecker delta function. The master equation (A 9) can be solved analytically using standard techniques (Gardiner 1983). 


\section{A.2. Steady-state behaviour}

One way of solving the forward master equations (A 9)-(A 10) makes use of the probability generating function (Gardiner 1983)

$$
G(z, t)=\sum_{n=0}^{\infty} z^{n} P(n ; t)
$$

which makes it possible to transform the master equations into a single partial differential equation. Multiplying equations (3.1)-(A 10) by $z^{n}$ and summing over $n$, we obtain

$$
\frac{\partial}{\partial t} G(z, t)=L_{z}[G]
$$

with $L_{z}[G]=\lambda^{\prime}(z-1)+\left\{\sigma+\mu z^{2}-(\mu+\sigma) z\right\} \frac{\partial}{\partial z} G$.

We have introduced the linear operator $L_{z}[\tilde{G}]$ (acting on $z$ ) for subsequent use in $\S$ A.3 (see the supplementary material for further information). Solving (A 13) and making use of the initial condition (A 11), we find that for $\mu>0$, the generating function takes the form

$$
G\left(z, t \mid N_{0}=n\right)=\left(\frac{\sigma-\mu}{(C \mu-\mu) z+\sigma-C \mu}\right)^{n+\lambda^{\prime} / \mu}\left(\frac{(C \sigma-\mu) z+\sigma(1-C)}{\sigma-\mu}\right)^{n}
$$

where $C=\mathrm{e}^{-t(\sigma-\mu)}$ (function $C$ corresponds to the autocorrelation function for flows at equilibrium), while for $\mu=0$

$$
G\left(z, t \mid N_{0}=n\right)=[1-C(1-z)]^{n} \exp \left[\frac{\lambda^{\prime}}{\sigma}(z-1+C(1-z))\right]
$$

where $C=\mathrm{e}^{-\sigma t}$. Two steady-state behaviours emerge from the generating function in the limit of $t \rightarrow \infty$ (as $\sigma>\mu$, we have $C \rightarrow 0$ )

$$
G_{s}(z)=\left(\frac{\sigma-\mu}{\sigma-\mu z}\right)^{\lambda^{\prime} / \mu},
$$

which turns out to be the probability generating function of the negative binomial distribution

$$
P_{s}(n)=\operatorname{NegBin}\left(n ; r_{n b}, p\right)=\frac{\Gamma(r+n)}{\Gamma\left(r_{n b}\right) n !} p^{r_{n b}}(1-p)^{n}, \quad n=0,1, \ldots,
$$

with $r_{n b}=\lambda^{\prime} / \mu$ and $p=1-\mu / \sigma$, and where $\Gamma$ denotes the gamma function. The mean is

$$
\langle N\rangle=\frac{\lambda^{\prime}}{\sigma-\mu},
$$

and the variance is

$$
\operatorname{var} N=\frac{\lambda^{\prime} \sigma}{(\sigma-\mu)^{2}}
$$


For $\mu=0$, we obtain the Poissonian solution $G_{s}(z)=\mathrm{e}^{-\lambda^{\prime}(z-1) / \sigma}$, corresponding to the Poisson distribution of rate $r_{p}=\lambda^{\prime} / \sigma$,

$$
P_{s}(n)=\frac{\left(r_{p}\right)^{n}}{n !} \mathrm{e}^{-r_{p}}
$$

$n=0,1, \ldots$. We retrieve the behaviour expected under Einstein's theory (Ancey et al. 2006): the sporadic motion of each particle is described as a Bernoulli process, and as the sum of $N$ Bernoulli processes is also a binomial process, $N$ behaves like a binomial variable. Recall that the binomial distribution converges towards the Poisson distribution when $n \rightarrow \infty$ while the product $n p$ remains fixed. Therefore, the Poisson distribution with parameter $n p$ can be used as an approximation to the binomial distribution $\operatorname{Bin}(n, p)$ provided that $n$ is sufficiently large and $p$ is sufficiently small.

It is worth noting that despite appearances, the solutions corresponding to the $\mu>$ 0 and $\mu=0$ cases are connected. Indeed, the negative binomial distribution can be interpreted as a compound probability distribution where the mixing distribution of the Poisson rate is a gamma distribution

$$
P_{s}(n)=\int_{a} \frac{\mathrm{e}^{-a} a^{n}}{n !} G a(a ; \alpha, \beta) \mathrm{d} a=\operatorname{NegBin}\left(n, r_{n b}, p\right)=\frac{\Gamma\left[r_{n b}+n\right]}{\Gamma\left[r_{n b}\right]} p^{r_{n b}}(1-p)^{n},
$$

with $\alpha=r_{n b}=\lambda^{\prime} / \mu$ and $\beta=1 / p-1=\mu /(\sigma-\mu)$. So, interestingly, we retrieve the Poisson distribution even in the case $\mu>0$, but hidden behind the stochastic variations of its rate $\lambda^{\prime}$. A physical interpretation can then be put forward. In the absence of collective entrainment $(\mu=0)$, the behaviour is Poissonian with a fixed rate $r_{p}=$ $\lambda^{\prime} / \sigma$ dictated by entrainment and deposition of individual particles. When collective entrainment occurs $(\mu>0)$, the behaviour can still be seen as Poissonian, but with a random rate. This random rate follows a gamma distribution, whose parameters are greatly influenced by $\mu$, especially when $\mu \rightarrow \sigma(\beta \rightarrow \infty)$. This observation prompts us to seek a generalized solution to the forward master equations (3.1)-(A 10) in the form of a Poisson representation.

\section{A.3. Poisson representation}

Following Gardiner (1983), we assume that we can expand $P(n, t)$ as a superposition of Poisson distributions

$$
P(n, t)=\int_{\mathscr{C}} \frac{\mathrm{e}^{-a} a^{n}}{n !} f(a, t) \mathrm{d} a,
$$

where the integration is made over a domain $\mathscr{C}$ to be specified and $f(a)$ is an integrable function. This representation can be seen as a Laplace-like transformation that allows us to pass from a discrete probability space, referred to as the $n$-space, to a continuous probability space, hereafter called the $a$-space. In general, we cannot take for granted that the function $f$ is a genuine probability density function. Indeed, the existence and uniqueness of $f$ can be shown, the constraint $\int_{\mathscr{C}} f(a) \mathrm{d} a=1$ is satisfied, but it cannot be demonstrated that $f$ is always positive. In the present case, we will show that such a condition is systematically and so, $f$ is a genuine probability density function. The Poisson representation technique is thus just a means to deal with probabilities in another space, allowing us to pass from discrete to continuous 
probabilities, which will make analytical calculations much easier. Moreover, the Poisson representation capitalizes on many interesting properties, the most important being that the forward master equations (3.1)-(A 10) in the $n$-space are represented by a Fokker-Planck equation in the $a$-space. Another interesting feature is that the moments in the $n$ - and $a$-spaces are related. Indeed, it can be easily checked that the $p$-factorial moment of $n$ is equal to $p$-moment of $a$

$$
\begin{aligned}
\langle n(n-1) \ldots(n-p+1)\rangle & =\sum_{n=0}^{\infty} n(n-1) \ldots(n-p+1) P(n, t) \\
& =\int_{\mathscr{C}} a^{p} f(a) \mathrm{d} a=\left\langle a^{p}\right\rangle,
\end{aligned}
$$

which implies that $\langle n\rangle=\langle a\rangle$ and $\left\langle n^{2}\right\rangle=\left\langle a^{2}\right\rangle+\langle a\rangle$ (see the supplementary material for the proof). This property will be quite useful below when mapping the $a$ - and $n$-spaces.

Let us now see in more detail what the Poisson representation tells us. The generating function (A 12) can be written as

$$
G(z, t)=\int_{\mathscr{C}} \exp [a(z-1)] f(a, t) \mathrm{d} a=\{K, f\},
$$

where the braces represent the inner product and $K$ the Laplace kernel

$$
\{f, g\}=\int_{\mathscr{C}} f(a) g(a) \mathrm{d} a, \quad K(z, a)=\exp [a(z-1)],
$$

for any functions $f$ and $g$ in the $a$-space. In the supplementary material, we show that the initial differential problem (A 13)

$$
\frac{\partial G}{\partial t}=L_{z}[G] \quad \text { with } G(z, t)=\{K(z, a), f(a, t)\},
$$

is equivalent to the following differential problem

$$
\left\{K, \frac{\partial f}{\partial t}\right\}=\left\{K, M_{a}^{*}[f]\right\}
$$

with

$$
M_{a}^{*}[f]=\mu \frac{\partial^{2} a f}{\partial a^{2}}-\frac{\partial}{\partial a}\left[\left(\lambda^{\prime}-a(\sigma-\mu)\right) f\right] .
$$

The governing equation for $f$ is then

$$
\frac{\partial f}{\partial t}=\mu \frac{\partial^{2} a f}{\partial a^{2}}-\frac{\partial}{\partial a}\left[\left(\lambda^{\prime}-a(\sigma-\mu)\right) f\right] .
$$

This is a second-order nonlinear parabolic diffusion equation, which has the same structure as that of a Fokker-Planck equation. This opens up interesting avenues for further use and interpretation (see § A.4). The solutions to (A 28) have been studied by Feller (1951). Equation (A 28) has also been used in economics to model shortterm interest rates (Cox, Ingersoll \& Ross 1985). Note that (A 28) is singular at $a=$ 0 (transformation from a parabolic to a hyperbolic problem). Feller (1951) showed 
that for $\lambda^{\prime}<\mu$, the problem is nearly regular and solutions with different boundary conditions can be determined. For $\mu<\lambda^{\prime}$, there exists a positivity and norm preserving solution of the initial value problem such that both it and its flux vanish at $a=0$. This means that $a=0$ acts both as an absorbing and a reflecting barrier. If the former condition is met $\left(\lambda^{\prime}<\mu\right), f$ tends to infinity when $a \rightarrow 0$, which means that $a=0$ corresponds to the highest probabilities (mode of $f$ ), but is never reached. In the latter case, $f(a, t)=0$, the mode of $f$ is somewhere within $(0, \infty)$. In all cases encountered here $\left(\sigma-\mu>0\right.$ and $\left.\lambda^{\prime}>0\right)$, the solution is a genuine probability density function (a non-central chi-squared distribution) (Cox et al. 1985). In the long term (when $\partial_{t} f=0$ ), when integrating (A 28), we retrieve the gamma distribution $f(a)=G a(a ; \alpha, \beta)$ with parameters $\alpha=\lambda^{\prime} / \mu>0$ and $\beta=\mu /(\sigma-\mu)>0$.

\section{A.4. Langevin representation}

As (A 28) takes the form of a Fokker-Planck equation with drift and diffusion functions $A(a)=\lambda^{\prime}+a(\mu-\sigma)$ and $D(a)=2 \mu a$, we can also interpret the process in terms of a Langevin equation for $a(t)$ :

$$
\mathrm{d} a=A \mathrm{~d} t+\sqrt{D} \mathrm{~d} W(t)=\left(\lambda^{\prime}-a(\sigma-\mu)\right) \mathrm{d} t+\sqrt{2 \mu a} \xi(t) \mathrm{d} t,
$$

where $\xi=\mathrm{d} W(t) / \mathrm{d} t$ represents the increments of a Wiener process $W(t)$ (white noise) satisfying $\langle\xi(t)\rangle=0$ and $\left\langle\xi(t) \xi\left(t^{\prime}\right)\right\rangle=\delta\left(t-t^{\prime}\right)$. Note the amplitude of the noise term is modulated by the collective entrainment parameter $\mu$. In the absence of collective entrainment $(\mu=0$ then $D=0)$, the process is purely deterministic in the $a$-space and we have

$$
\frac{\mathrm{d} a}{\mathrm{~d} t}=\lambda^{\prime}-a(\sigma-\mu) \Rightarrow a(t)=\frac{\lambda^{\prime}}{\sigma-\mu}+a_{0} \exp [-(\sigma-\mu) t] .
$$

When collective entrainment occurs $(\mu>0)$, the process is random, with a multiplicative noise term. In that case, the solution to (A 29) subject to $a(0)=a_{0}$ is

$$
a(t)=a_{\infty}+\left(a_{0}-a_{\infty}\right) \mathrm{e}^{-\kappa t}+\sqrt{2 \mu} \mathrm{e}^{-\kappa t} \int_{0}^{t} \mathrm{e}^{\kappa t^{\prime}} \sqrt{a\left(t^{\prime}\right)} \mathrm{d} W\left(t^{\prime}\right),
$$

with $\kappa=\sigma-\mu$ and where $a_{\infty}=\langle a\rangle_{s}=\lambda^{\prime} /(\sigma-\mu)$ denotes the mean solution in a steady state. We then deduce the expectation

$$
\langle a(t)\rangle=a_{\infty}+\left(a_{0}-a_{\infty}\right) \mathrm{e}^{-\kappa t} .
$$

After integration and a few algebraic manipulations (see the supplementary material), we find that the variance is

$$
\operatorname{var} a(t)=2 \frac{\mu}{\kappa} a_{0} \mathrm{e}^{-2 \kappa t}\left(\mathrm{e}^{\kappa t}-1\right)+\frac{\mu}{\kappa} a_{\infty} \mathrm{e}^{-2 \kappa t}\left(\mathrm{e}^{\kappa t}-1\right)^{2} .
$$

The covariance for $t>s$ is

$$
\operatorname{cov}(a(s), a(t))=\langle(a(s)-\langle a\rangle)(a(t)-\langle a\rangle)\rangle=\mathrm{e}^{-\kappa(t-s)} \operatorname{var} a(s),
$$

which shows that the autocorrelation function is

$$
C(\tau)=\frac{\operatorname{cov}(a(s), a(s+\tau))}{\operatorname{var} a(s)}=\mathrm{e}^{-\tau / t_{c}},
$$

where $t_{c}=\kappa^{-1}$ denotes the autocorrelation time. 


\section{A.5. Implications for the number of particles or particle activity}

After this mathematical stroll in the $a$-space, let us highlight some key points. We started with a counting problem, in which we were interested in computing the evolution of $N$ particles in a control volume, knowing that particles can be entrained (through individual or collective entrainment mechanisms controlled by $\lambda^{\prime}$ and $\mu$, respectively) or deposited (at a rate $\sigma$ ). The governing equation for $N$ is given by the master equations (3.1)-(A 10). Instead of working in a discrete probability space, we can work in a continuous probability space, the $a$-space, without using any approximation or assumption such as the truncated Kramers-Moyal or system-size expansion that we used in an earlier paper (Ancey 2010). The resulting representation is thus strictly equivalent to the original master equation (3.1). In this space, the time-dependent response of the system or its steady-state solution can be computed exactly. Using the Poisson representation (A 22), we thus find again that in the $n$-space, the steady-state solution is the negative binomial distribution (3.2). Using the definition of the Poisson representation (A 22) or the relationships between the $a$ and $n$ moments, we can deduce the probability distribution function $P(n, t)$ and its moments $\left\langle n^{p}\right\rangle$ at any order $p$. For instance, the mean number of particles $\langle N\rangle$ in $\mathscr{V}$ is

$$
\frac{\mathrm{d}}{\mathrm{d} t}\langle N\rangle=\lambda^{\prime}-\kappa\langle N\rangle,
$$

or in terms of the particle activity

$$
\frac{\mathrm{d}}{\mathrm{d} t}\langle\gamma(t)\rangle=\lambda-\kappa\langle\gamma(t)\rangle .
$$

We have also found that the steady state is approached for $t \gg t_{c}=\kappa^{-1}$ and when $\mu>0, a(t)$ comes close to a constant value $a_{\infty}=\lambda^{\prime} /(\sigma-\mu)$. However, the fluctuations prevent its attainment. Indeed, the corresponding distribution $f(a)$ in the $a$-space is the gamma distribution $G a(a ; \alpha, \beta)$ with $\alpha=\lambda^{\prime} / \mu$ and $\beta=\mu /(\sigma-\mu)$. When $\lambda^{\prime}<\mu<\sigma$, $G a(a ; \alpha, \beta)$ tends to infinity when $a \rightarrow 0$, which implies that there is a high probability that $a$ remains close to 0 without reaching it. When $0<\mu<\lambda^{\prime}$, the peak of probability is located at $a=(\alpha-1) \beta=\left(\lambda^{\prime}-\mu\right) /(\sigma-\mu)=a_{\infty}-\mu / \kappa$. In both cases, $a$ is expected to fluctuate with variable amplitude around the asymptotic value $a_{\infty}$.

Another interesting feature is revealed by the solution (A 30) to the Langevin equation (A 29): starting from its initial value, $a$ relaxes exponentially to the steady-state value $a_{\infty}$. In the absence of collective entrainment $(\mu=0)$, this value is reached asymptotically, which means that in the $n$-space, the distribution of $N$ being the Poisson distribution, the fluctuations are Poissonian and bounded $\left(\langle N\rangle=\operatorname{var} N=\lambda^{\prime} / \sigma\right)$. Collective entrainment adds noise to these Poissonian fluctuations: in (A 30), the strength of the noise term is $\sqrt{2 \mu a}$. A square-root nonlinear multiplicative noise term may amplify the fluctuations of $a$, thus giving rise to large and non-Poissonian fluctuations of $N$ in the $n$-space. From this perspective, the collective entrainment parameter $\mu$ controls the deviations from the Poissonian fluctuations. Note also that when $\mu \rightarrow \sigma$, the autocorrelation time $t_{c}$ becomes indefinitely long, which means that in practice, time series of sediment transport rates may exhibit bursts of activity over fairly long periods of time. Counterintuitively, though, the parameter $\mu$ has no effect on the coefficient of variation $\operatorname{var}^{1 / 2} N /\langle N\rangle=\sqrt{\sigma / \lambda^{\prime}}$. This coefficient is independent of $\mu$ and can become very large 
only when $\lambda^{\prime} \rightarrow 0$, which implies that fluctuations become much larger than the mean value for incipient sediment transport conditions.

All of these features demonstrate that, depending on the specific values taken by the parameters $\lambda^{\prime}, \sigma$ and $\mu$, a wide range of situations from well-behaved Poissonian fluctuations to wilder non-Poissonian fluctuations may be observed. The actual behaviour is therefore much richer than that exhibited by the deceptively simple mean-field equation (A 35) for the mean value $\langle N\rangle$.

\section{Appendix B. Sediment transport rate}

Using our definition of the sediment transport rate (2.2), we can calculate the instantaneous particle flux

$$
\dot{n}(t ; \mathscr{V})=\frac{1}{\Delta x} \sum_{i=1}^{N(t)} U_{p, i}
$$

where both $N$ and $U_{p, i}$ are random variables. For a stationary process, their probability distributions are given by (3.2) (or (3.5) if $\mu=0$ ) and (3.10), respectively. The probability density function of the sum of random variables drawn from the same distribution can be calculated by taking the Fourier transform of the convolution product, then inverting the result. After a little bit of work, we eventually find that

$$
P_{\dot{n}}(\dot{n})=P_{s}(0) \delta(\dot{n})+\frac{\zeta \Delta x}{\bar{u}_{s}} \sqrt{\frac{2}{\pi}} \sum_{k=1}^{\infty} P_{s}(k) \frac{\exp \left[-\zeta^{2}\left(\left(\dot{n} \Delta x-k \bar{u}_{s}\right)^{2} / 2 k \bar{u}_{s}^{2}\right)\right]}{\sqrt{k}(1+\operatorname{erf}(\sqrt{k} \zeta / \sqrt{2}))},
$$

where $P_{s}(k)$ is given by (3.2) if $\mu>0$ and (3.5) if $\mu=0$. Note that the probability density function of $\dot{n}$ is discontinuous at $\dot{n}=0$ : there is a finite probability $P_{s}(0)=(1-$ $\mu / \sigma)^{\lambda^{\prime} / \mu}$ for $\mu>0\left(P_{s}(0)=\exp \left(-\lambda^{\prime} / \sigma\right)\right.$ for $\left.\mu=0\right)$ that there is no moving particle within the window, in other words, that the particle flux is zero. Provided that there is at least one moving particle, $P_{\dot{n}}$ jumps to another finite value.

As the structure of $P_{\dot{n}}(\dot{n})$ in (B 2) is complicated, it is a priori difficult to draw conclusions about the part played by each parameter in the fluctuations of $\dot{n}$. Some remarkable features can, however, be deduced from numerical solutions and approximations. Figure 10 shows examples of variations of $P_{\dot{n}}(\dot{n})$ for $\zeta$ ranging from 0.5 to 5 . For low $\zeta$ values, the probability density function varies smoothly and slowly except for the point of discontinuity $\dot{n}=0$, as explained above. Increasing $\zeta$ leads to (i) a faster (but still exponential-like) decay at larger values of $\dot{n}$, which is little influenced by the actual value of $\zeta$, and (ii) the development of sharp peaks of probability for the lowest values of $\dot{n}$ : in a dilute flow much of the flux is carried by a couple of particles and, in the absence of velocity fluctuations, the particle flux exhibits this bumpy landscape in which each peak corresponds to the crossing of one particle.

Numerical estimations have also shown that $\zeta$ influences the moments of the probability density function $P_{\dot{n}}(\dot{n})$, but this influence is vanishingly small for $\zeta \geqslant 2$. Assuming that there is no correlation between the fluctuations of $N$ and $U$, we can deduce the moments of $\dot{n}$. For instance, making use of (3.3) and (3.11), we find that the steady-state particle flux can be approximated by

$$
\langle\dot{n}(t ; \mathscr{V})\rangle \approx \frac{1}{\Delta x}\langle N\rangle\langle u\rangle_{e q .}=\frac{\bar{u}_{s}}{\Delta x} \frac{\lambda^{\prime}}{\sigma-\mu} F_{e q .}(\zeta)
$$


Equivalently, we can use the volumetric transport rate defined by (2.1)

$$
\left\langle q_{s}(t ; \mathscr{V})\right\rangle \approx \frac{\varpi_{p}}{\Delta x}\langle N\rangle\langle u\rangle_{e q .}=\bar{u}_{s} \frac{\lambda}{\sigma-\mu} F_{e q .}(\zeta),
$$

whose square coefficient of variation is

$$
\frac{\operatorname{var} q_{s}}{\left\langle q_{s}\right\rangle^{2}} \approx\left(F_{e q .}(\zeta)+\frac{1}{\zeta^{2}}\right) \frac{1}{F_{e q .}^{2}(\zeta)} \frac{\sigma+\lambda^{\prime}}{\lambda^{\prime}}-1
$$

We fall back into the same discussions about the origins of fluctuations (see $\S$ A.5). Again, we find that large fluctuations (relative to the mean) are observed when $\zeta \rightarrow 0$ or $\lambda^{\prime} \rightarrow 0$, i.e. for flow conditions that correspond to incipient sediment motion. It is worth noting that although it controls the nature of the fluctuations, the collective entrainment parameter $\mu$ plays no part in determining the coefficient of variation. Collective entrainment has thus significant effects on the correlations and probability distributions of sediment transport rates, but does not influence the relative amplitude of the fluctuations. For flow conditions pertaining to intense sediment transport $(\zeta \gg 1), F_{e q}$. is close to unity, which implies that the square coefficient of variation is $\sigma / \lambda$, thus becoming independent of particle velocity fluctuations.

Although Einstein (1950) used probabilistic concepts to derive his bed load equation, he did not end up with a probability distribution for the sediment transport rate. As mentioned in $\S$ A.2, the arguments used by Einstein (1950) lead to a binomial variation of the number of moving particles, thus a Poisson distribution in the limit $p \rightarrow 0$ (i.e. $\mu \rightarrow \sigma$ ) and $N \gg 1$. This means that intense sediment transport exhibits bounded Poissonian fluctuations, with the coefficient of variation $\operatorname{var}^{1 / 2} q_{s} /\left\langle q_{s}\right\rangle=r_{p}^{-1 / 2}$ given by the steady-state Poisson distribution (3.5). Hamamori (1962) is credited with the first attempt to derive the probability distribution for the sediment transport rate. He considered that bed load transport rate fluctuations arise from the migration of bed forms. He obtained a non-parametric density distribution function of the bed load transport rate

$$
P\left(q_{s}\right)=\frac{1}{4\left\langle q_{s}\right\rangle} \log \left(4 \frac{\left\langle q_{s}\right\rangle}{q_{s}}\right),
$$

which implies that the fluctuations are bounded: $0<q_{s}<4\left\langle q_{s}\right\rangle$, and that the square coefficient of variation is constant: $\operatorname{var} q_{s} /\left\langle q_{s}\right\rangle^{2}=7 / 9$. More recently, Turowski (2010) used a two-parameter distribution derived from the normal distribution, called the Birnbaum-Saunders distribution

$$
P\left(q_{s}\right)=\frac{q_{s}+\alpha}{2 \beta q_{s} \sqrt{2 \pi \alpha q_{s}}} \exp \left[-\frac{\left(q_{s}-\alpha\right)^{2}}{2 \alpha \beta^{2} q_{s}}\right],
$$

with $\alpha$ and $\beta$ two calibration parameters. The mean value is $\left\langle q_{s}\right\rangle=\alpha\left(1+\beta^{2} / 2\right)$ and the coefficient of variation is found to range from 0 to $\sqrt{5}$. Figure 11 shows the comparison of the probability distributions (B 2), (B 6), (B 7) for a particular case. As the fluctuations are bounded, Hamamori's relation is unable to capture the exponential tail of the distribution and tends to overestimate the bed load transport rate significantly (compared with what (B 2) predicts) in the limit of $\dot{n} \rightarrow 0$. This latter shortcoming is also observed for the Birnbaum-Saunders distribution, but the tail behaviour is consistent with that predicted by our model (B 2). On the whole, the 


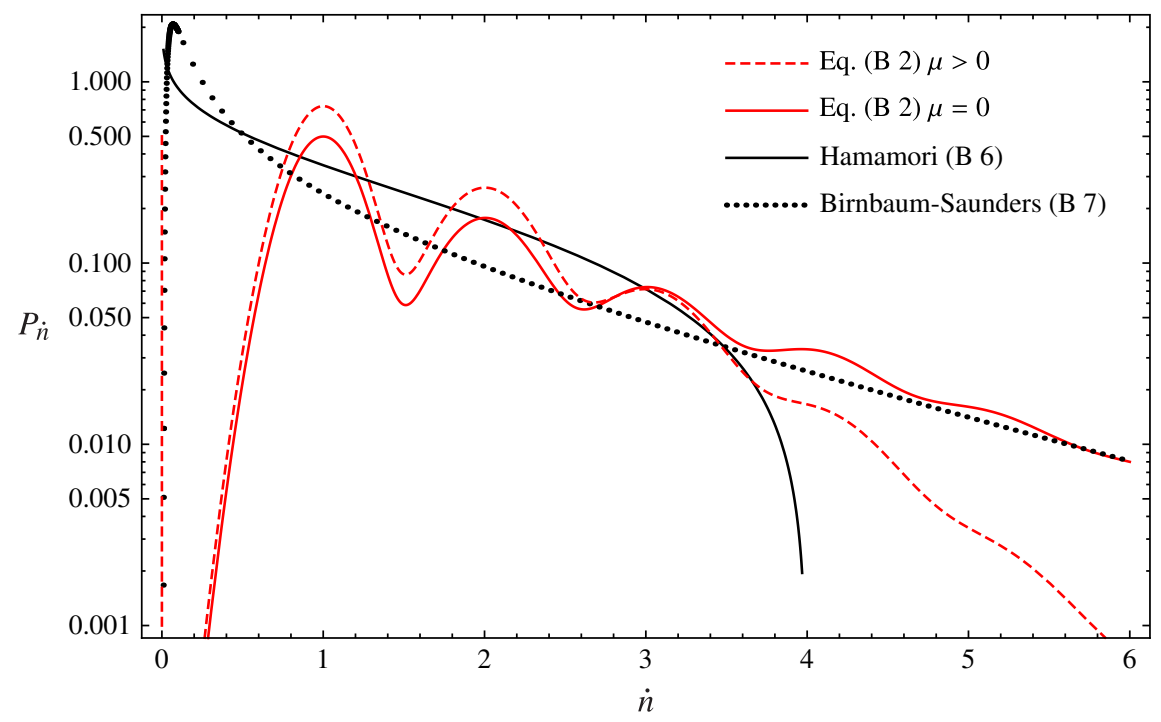

Figure 11. (Colour online) Comparison of the probability density function $P(\dot{n})$ in a $\log$ linear plot: Hamamori's equation (B 6) (with $\langle\dot{n}\rangle=1$ bead $\mathrm{s}^{-1}$ ) and Birnbaum-Saunders distribution (B 7) (with $\alpha=0.451$ and $\beta=1.556$ ). We also report the probability density function $P_{\dot{n}}(\dot{n})$ for $\zeta=5\left(\Delta x=1 \mathrm{~m}, \bar{u}_{s}=1 \mathrm{~m} \mathrm{~s}^{-1}\right)$ : when the number of moving particles follows the negative binomial distribution (3.2) (solid (red online) line) with $r=1\left(\lambda^{\prime}=\mu\right)$ and $p=0.5\left(\sigma=2 \lambda^{\prime}\right)$ or the Poisson distribution (3.5) (dashed (red online) line) with $r_{p}=1$ bead $\mathrm{s}^{-1}$. Except for the Poisson distribution (whose variance equals the mean), the coefficient of variation is $\sqrt{2}$ and all of the distributions have the same mean $(\langle\dot{n}\rangle=$ 1 bead $\mathrm{s}^{-1}$ ).

general impression one gets from figure 10 is that the Birnbaum-Saunders distribution smooths out the ups and downs in the probability distribution (B 2). Although the point of this paper is not to discuss the agreement with field and experimental data, note that the high-resolution data confirm (i) the significant proportion of zero values of the particle flux and (ii) the highly fluctuating nature of time series, two features that are consistently described by (B 2) and figure 11 (Ancey et al. 2006, 2008; Radice 2009; Singh et al. 2009).

\section{REFERENCES}

Allen, E. J. 2007 Modeling with Itô Stochastic Differential Equations. Springer.

ANCEY, C. 2010 Stochastic approximation of the Exner equation under lower-regime conditions. J. Geophys. Res. 115, F00A11.

Ancey, C., Bigillon, F., Frey, P., LAnier, J. \& Ducret, R. 2002 Saltating motion of a bead in a rapid water stream. Phys. Rev. E 66, 036306.

Ancey, C., BöHM, T., JodeAU, M. \& Frey, P. 2006 Statistical description of sediment transport experiments. Phys. Rev. E 74, 011302.

Ancey, C., Davison, A. C., Böhm, T., Jodeau, M. \& Frey, P. 2008 Entrainment and motion of coarse particles in a shallow water stream down a steep slope. J. Fluid Mech. 595, 83-114.

Ballio, F., Nikora, V. \& Coleman, S. E. 2013 On the definition of solid discharge in hydroenvironment research and applications. J. Hydraul. Res. in press. 
Balmforth, N. J. \& Provenzale, A. 2001 Patterns of dirt. In Geomorphological Fluid Mechanics (ed. N. J. Balmforth \& A. Provenzale), pp. 369-393. Springer.

Böhm, T., Ancey, C., Frey, P., Reboud, J.-L. \& Duccotet, C. 2004 Fluctuations of the solid discharge of gravity-driven particle flows in a turbulent stream. Phys. Rev. E 69, 061307.

Bohorquez, P. \& DARby, S. E. 2008 The use of one- and two-dimensional hydraulic modelling to reconstruct a glacial outburst flood in a steep Alpine valley. J. Hydrol. 361, 240-261.

Bulut, U. \& Allen, E. J. 2012 Derivation of SPDEs for correlated random walk transport models in one and two dimensions. Stoch. Anal. Appl. 30, 553-567.

BUnte, K. \& ABT, S. 2005 Effect of sampling time on measured gravel bed load transport rates in a coarse-bedded stream. Water Resour. Res. 41, W11405.

Campagnol, J., Radice, A. \& Ballio, F. 2012 Scale-based statistical analysis of sediment fluxes. Acta Geophys. 60, 1744-1777.

CAO, Z., DAY, R. \& Egashira, S. 2002 Coupled and decoupled numerical modeling of flow and morphological evolution in alluvial rivers. J. Hydraul. Eng. 128, 306-321.

Charru, F. 2011 Hydrodynamic Instabilities. Cambridge University Press.

Chatanantavet, P., Whipple, K. X., Adams, M. A. \& Lamb, M. P. 2013 Experimental study on coarse grain saltation dynamics in bedrock channels. J. Geophys. Res. F118, 1161-1176.

CHurch, M. 2006 Bed material transport and the morphology of alluvial river channels. Annu. Rev. Earth Planet. Sci. 34, 325-354.

Coleman, S. E. \& Nikora, V. I. 2009 Bed and flow dynamics leading to sediment-wave initiation. Water Resour. Res. 45, W04402.

Colombini, M. \& Stocchino, A. 2011 Ripple and dune formation in rivers. J. Fluid Mech. 673, $121-131$.

Colombini, M. \& Stocchino, A. 2012 Three-dimensional river bed forms. J. Fluid Mech. 695, 63-80.

Cox, J. C., Ingersoll, J. E. \& Ross, S. A. 1985 A theory of the term structure of interest rates. Econometrica 53, 385-407.

Cudden, J. R. \& Hoey, T. B. 2003 The causes of bedload pulses in a gravel channel: the implications of bedload grain-size distributions. Earth Surf. Process. Landf. 28, 1411-1428.

Cui, Y., Parker, G., Lisle, T. E., Pizzuto, J. E. \& Dodd, A. M. 2005 More on the evolution of bed material waves in alluvial rivers. Earth Surf. Process. Landf. 30, 107-114.

DefinA, A. 2000 Two-dimensional shallow flow equations for partially dry areas. Water Resour. Res. 36, 3251-3264.

Dogan, E. \& Allen, E. J. 2011 Derivation of stochastic partial differential equations for reactiondiffusion processes. Stoch. Anal. Appl. 29, 424-443.

Dornic, I., Chate, H. \& Munoz, M. A. 2005 Integration of Langevin equations with multiplicative noise and the viability of field theories for absorbing phase transitions. Phys. Rev. Lett. 94, 100601.

Einstein, H. A. 1950 The bed-load function for sediment transportation in open channel flows. Tech. Rep. Technical Report No. 1026. United States Department of Agriculture.

Fasolato, G., Ronco, P., Langendoen, E. J. \& Di Silvio, G. 2011 Validity of uniform flow hypothesis in one-dimensional morphodynamic models. J. Hydraul. Eng. 137, 183-195.

FELler, W. 1951 Two singular diffusion problems. Ann. Maths. 54, 173-182.

FERGUSON, R. I. 2012 River channel slope, flow resistance, and gravel entrainment thresholds. Water Resour. Res. 48, W05517.

Ferguson, R. I. \& Church, M. 2009 A critical perspective on 1-D modeling of river processes: gravel load and aggradation in lower Fraser River. Water Resour. Res. 45, W11424.

Fowler, A. C. 1997 Mathematical Models in the Applied Sciences. Cambridge University Press.

Fowler, A. C., Kopteva, N.\& OAKLEy, C. 2007 The formation of river channels. SIAM J. Appl. Math. 67, 1016-1040.

Furbish, D. J., Haff, P. K., Roseberry, J. C. \& Schmeeckle, M. W. $2012 a$ A probabilistic description of the bed load sediment flux: 1. Theory. J. Geophys. Res. 117, F03031. 
Furbish, D. J., Roseberry, J. C. \& Schmeeckle, M. W. $2012 b$ A probabilistic description of the bed load sediment flux: 3. The particle velocity distribution and the diffusive flux. J. Geophys. Res. 117, F03033.

GANTI et al., 2010.

García, M. H. 2007 Sediment transport and morphodynamics. In Sedimentation Engineering (ed. M. H. García), ASCE Manuals and Reports on Engineering Practice, vol. 110, pp. 21-164. American Society of Civil Engineers.

Gardiner, C. W. 1983 Handbook of Stochastic Methods. Springer.

Gardiner, C. W. \& Chaturvedi, S. 1977 The Poisson representation. I. A new technique for chemical master equations. J. Stat. Phys. 17, 429-468.

Gillespie, D. T. 1992 Markov Processes: An Introduction for Physical Scientists. Academic Press.

Gillespie, D. T. 2007 Stochastic simulation of chemical kinetics. Annu. Rev. Phys. Chem. 58, 35-55.

Gomez, B. 1991 Bedload transport. Earth. Sci. Rev. 31, 89-132.

GRAF, W. H. \& AltinakAR, S. 2005 Transport of sediments. In Encyclopedia of Hydrological Sciences (ed. M. G. Andersen), John Wiley \& Sons.

Hamamori, A. 1962 A theoretical investigation on the fluctuations of bed load transport. Tech. Rep. Report R4. Delft Hydraulics Laboratory.

Helbing, D. 2001 Traffic and related self-driven many-particle systems. Rev. Mod. Phys. 73, 10671141.

Heyman, J., Mettra, F., Ma, H. B. \& Ancey, C. 2013 Statistics of bedload transport over steep slopes: separation of time scales and collective motion. Geophys. Res. Lett. 40, 128-133.

Hita, J. L. \& ORTIZ de ZÁRATE, J. M. 2013 Spatial correlations in nonequilibrium reaction-diffusion problems by the Gillespie algorithm. Phys. Rev. E 87, 052802.

Hoyle, R. 2006 Pattern Formation. Cambridge University Press.

IACUS, S. M. 2008 Simulation and Inference for Stochastic Differential Equations. Springer.

Jerolmack, D. \& Mohrig, D. 2005 A unified model for subaqueous bed form dynamics. Water Resour. Res. 41, W12421.

Katul, G., Wiberg, P., Albertson, J. \& Hornberger, G. 2002 A mixing layer theory for flow resistance in shallow streams. Water Resour. Res. 38, 1250-1258.

Lajeunesse, E., Malverti, L. \& Charru, F. 2010 Bed load transport in turbulent flow at the grain scale: experiments and modeling. J. Geophys. Res. 115, F04001.

LANZONI, S. 2008 Mathematical modelling of bedload transport over partially dry areas. Acta Geophys. 56 (3), 734-752.

Lisle, T. E., Cui, Y., Parker, G., Pizzuto, J. E. \& Dodd, A. 2001 The dominance of dispersion in the evolution of bed material waves in gravel-bed rivers. Earth Surf. Process. Landf. 26, $1409-1420$.

Logan, J. D. 2001 Transport Modeling in Hydrogeochemical Systems. Springer.

Martin, R. L., Jerolmack, D. J. \& SChUmer, R. 2012 The physical basis for anomalous diffusion in bed load transport. J. Geophys. Res. 117, F01018.

Méndez, V., Fedotov, S. \& Horsthemke, W. 2010 Reaction-Transport Systems: Mesoscopic Foundations, Fronts, and Spatial Instabilities. Springer.

MinieR, J.-P. \& PEIRANO, E. 2001 The pdf approach to turbulent polydispersed two-phase flows. Phys. Rep. 352, 1-214.

Niño, Y., Atala, A., Barahona, M. \& ARacena, D. 2002 Discrete particle model for analyzing bedform development. J. Hydraul. Eng. 128, 381-389.

PAOla, C. 2000 Quantitative models of sedimentary basin filling. Sedimentology 47, 121-178.

PaOla, C. \& Voller, V. R. 2005 A generalized Exner equation for sediment mass balance. J. Geophys. Res. 110, F04014.

PARÉS, C. 2006 Numerical methods for nonconservative hyperbolic systems: a theoretical framework. SIAM J. Numer. Anal. 44, 300-321.

PARKer, G. 2008 Transport of gravel and sediment mixtures. In Sedimentation Engineering: Processes, Measurements, Modeling, and Practice (ed. M. Garcìa), pp. 165-252. ASCE. 
PARKer, G. \& IzUmi, N. 2000 Purely erosional cyclic and solitary steps created by flow over a cohesive bed. J. Fluid Mech. 419, 203-238.

Parker, G., PaOla, C. \& Leclair, S. 2000 Probabilistic Exner sediment continuity equation for mixtures with no active layer. J. Hydraul. Eng. 126, 818-826.

Powell, D. M. 1998 Patterns and processes of sediment sorting in gravel-bed rivers. Prog. Phys. Geogr. 22, 1-32.

RADICE, A. 2009 Use of the Lorenz curve to quantify statistical nonuniformity of sediment transport rate. J. Hydraul. Eng. 135, 320-326.

Radice, A., Ballio, F. \& Nikora, V. 2009 On statistical properties of bed load sediment concentration. Water Resour. Res. 45, W06501.

RECKING, A. 2013 An analysis of nonlinearity effects on bed load transport prediction. J. Geophys. Res. 118, 20090.

Recking, A., Frey, P., Paquier, A., Belleudy, P. \& Champagne, J. Y. 2008 Feedback between bed load transport and flow resistance in gravel and cobble bed rivers. Water Resour. Res. 44, W05412.

Recking, A., Liébault, F., Peteuil, C. \& Jolimet, T. 2012 Testing bedload transport equations with consideration of time scales. Earth Surf. Process. Landf. 37, 774-789.

Rogers, S. S., Waigh, T. A., ZhaO, X. \& LU, J. R. 2007 Precise particle tracking against a complicated background: polynomial fitting with Gaussian weight. Phys. Biol. 4, 220-227.

Roseberry, J. C., SChmeeckle, M. W. \& Furbish, D. J. 2012 A probabilistic description of the bed load sediment flux: 2. Particle activity and motions. J. Geophys. Res. 117, F03032.

Sagués, F., SAncho, J. M. \& García-Ojalvo, J. 2007 Spatiotemporal order out of noise. Rev. Mod. Phys. 79, 829-882.

SCHUlz, M. 2008 Chemical reactions and fluctuations: exact substitute processes for diffusion-reaction systems with exclusion rules. Eur. Phys. J. Spec. Top. 161, 150-153.

Seminara, G. 2010 Fluvial sedimentary patterns. Annu. Rev. Fluid Mech. 42, 43-66.

Singh, A., Fienberg, K., Jerolmack, D. J., Marr, J. \& Foufoula-Georgiou, E. 2009 Experimental evidence for statistical scaling and intermittency in sediment transport rates. J. Geophys. Res. 114, 2007JF000963.

TIPPER, J. C. 2007 The 'stochastic river': the use of budget-capacity modelling as a basis for predicting long-term properties of stratigraphic successions. Sediment Geol. 202, 269-280.

Turowski, J. M. 2010 Probability distributions of bed load transport rates: a new derivation and comparison with field data. Water Resour. Res. 46, W08501.

Vollmer, S. \& Kleinhans, M. G. 2007 Predicting incipient motion, including the effect of turbulent pressure fluctuations in the bed. Water Resour. Res. 43, W05410.

Wiberg, P. L. \& SMith, J. D. 1985 A theoretical model for saltating grains in water. J. Geophys. Res. C 90, 7341-7354.

Wilcock, P. R., Pitlick, J. \& CUI, Y. 2009 Sediment transport primer: estimating bed-material transport in gravel-bed rivers. Tech. Rep. US Department of Agriculture, Forest Service, Rocky Mountain Research Station.

Yalin, M. S. 1972 Mechanics of Sediment Transport. Pergamon Press.

ZHANG et al., 2012. 